Homology, Homotopy and Applications, vol.22(2), 2020, pp.181-202

\title{
KOSZUL DUALITY AND THE HOCHSCHILD COHOMOLOGY OF ARTIN-SCHELTER REGULAR ALGEBRAS
}

\author{
LEILEI LIU \\ (communicated by Charles A. Weibel)
}

\begin{abstract}
We identify two Batalin-Vilkovisky algebra structures, one obtained by Kowalzig and Krahmer on the Hochschild cohomology of an Artin-Schelter regular algebra with semisimple Nakayama automorphism and the other obtained by Lambre, Zhou and Zimmermann on the Hochschild cohomology of a Frobenius algebra also with semisimple Nakayama automorphism, provided that these two algebras are Koszul dual to each other.
\end{abstract}

\section{Introduction}

In 2014, Kowalzig and Krahmer showed in [12] that the Hochschild cohomology of an Artin-Schelter (AS for short) regular algebra with semisimple Nakayama automorphism has a Batalin-Vilkovisky algebra structure. Soon after that, Lambre, Zhou and Zimmerman proved in [14] that the Hochschild cohomology of a Frobenius algebra with semisimple Nakayama automorphism also admits a Batalin-Vilkovisky algebra structure. These two Batalin-Vilkovisky algebras are nontrivial in the sense that the corresponding Batalin-Vilkovisky operators in both cases generate the Gerstenhaber bracket on the Hochschild cohomology. In this paper, we identify these two BatalinVilkovisky algebra structures, provided that these two algebras are Koszul dual to each other. Let us start with some background.

In 1998, Van den Bergh introduced in [23] the "noncommutative Poincaré duality" for associative algebras. As a corollary, one obtains that for an AS-regular algebra of global dimension $n$, say $A$, there is an isomorphism between the Hochschild cohomology of $A$ and the Hochschild homology of $A$ with coefficients in $A_{\sigma}$

$$
\mathrm{HH}^{\bullet}(A) \cong \mathrm{HH}_{n-\bullet}\left(A ; A_{\sigma}\right),
$$

where $\sigma$ is the Nakayama automorphism of $A$ (see loc. cit. Proposition 2). Now if we assume $\sigma$ is semisimple, then it was proved in $[\mathbf{1 2}]$ that $\mathrm{HH}_{\bullet}\left(A ; A_{\sigma}\right)$ can be computed by a subcomplex of the corresponding Hochschild complex on which the Connes cyclic operator exists. Therefore we may pull back the Connes cyclic operator to $\mathrm{HH}^{\bullet}(A)$ via (1), which is usually denoted by $\Delta$. They showed $\Delta$ generates the Gerstenhaber bracket on $\mathrm{HH}^{\bullet}(A)$, which means $\left(\mathrm{HH}^{\bullet}(A), \cup,\{-,-\}, \Delta\right)$ is a Batalin-Vilkovisky algebra.

Received January 25, 2019, revised June 11, 2019, October 14, 2019; published on April 29, 2020. 2010 Mathematics Subject Classification: 14A22, 16E40, 16 S38.

Key words and phrases: Artin-Schelter algebra, Koszul duality, cohomology, Batalin-Vilkovisky algebra.

Article available at http://dx.doi.org/10.4310/HHA.2020.v22.n2.a12

Copyright (C) 2020, International Press. Permission to copy for private use granted. 
In [14], it was proved that a Frobenius algebra with semisimple Nakayama automorphism, say $A^{!}$, has a Batalin-Vilkovisky algebra structure on $\mathrm{HH}^{\bullet}\left(A^{!}\right)$.

To relate these two Batalin-Vilkovisky algebra structures, let us recall a result of P. Smith. In [18, Proposition 5.10] he showed that for a graded connected Koszul algebra $A, A$ is AS-regular if and only if its Koszul dual $A^{!}$is Frobenius. Buchweitz showed (see $[\mathbf{2}]$ ), there is an isomorphism

$$
\mathrm{HH}^{\bullet}(A) \cong \mathrm{HH}^{\bullet}\left(A^{!}\right)
$$

as Gerstenhaber algebras. So it is natural to ask for an AS-regular algebra $A$ with semisimple Nakayama automorphism whether the above isomorphism is an isomorphism of Batalin-Vilkovisky algebras. In this paper we give an affirmative answer to this question:

Theorem 1.1. Suppose $A$ is a Koszul AS-regular algebra with semisimple Nakayama automorphism. Denote by $A^{!}$its Koszul dual algebra. Then

$$
\mathrm{HH}^{\bullet}(A) \cong \mathrm{HH}^{\bullet}\left(A^{!}\right)
$$

as Batalin-Vilkovisky algebras.

This paper can be viewed as a sequel to [5], where the isomorphism of BatalinVilkovisky algebras on two Hochschild cohomology groups are proved for Koszul Calabi-Yau algebras, verifying a conjecture of Rouquier given in the preprint [8] of Ginzburg.

Note that Calabi-Yau algebras and AS-regular algebras are highly related: in [19], Reyes, Rogalski and Zhang introduced the notion of twisted Calabi-Yau algebras (a Calabi-Yau algebra is twisted with trivial twisting), and proved that an algebra is twisted Calabi-Yau if and only if it is AS-regular (see also [25] for some partial result). Thus the isomorphism of Batalin-Vilkovisky algebras for Koszul Calabi-Yau algebras, proved in [5], is a special case of Theorem 1.1. In other words, we may view Theorem 1.1 as a twisted version of Rouquier's conjecture.

\section{Acknowledgments}

I would like to thank Xiaojun Chen and Farkhod Eshmatov for many helpful conversations and encouragements. This work is partially supported by NSFC (Nos. 11521061 and 11671281).

Notation. Throughout this paper, $k$ denotes a field of character 0 . All tensors and Homs are over $k$ unless otherwise specified. All algebras (resp. coalgebras) are unital and augmented, (resp. co-unital and co-augmented) over $k$. If $A$ is an associative algebra, then $A^{o p}$ is its opposite and $A^{e}=A \otimes A^{o p}$ is its envelope. Suppose $V_{\bullet}$ is a graded vector space, then the shift of the grading of $V_{\bullet}$ down by $n$ is denoted by $s^{-n} V_{\bullet}$ or $V_{\bullet}[n]$, i.e., $\left(S^{-n} V_{\bullet}\right)_{m}=V_{n+m}$ and $\left(V_{\bullet}[n]\right)_{m}=V_{m+n}$.

\section{Preliminaries on Hochschild homology}

In this section, we recall the Hochschild homology and cohomology of associative algebras. These two homology groups, together with algebraic operations on them, 
form a so-called differential calculus, a notion introduced by Tamarkin and Tsygan in $[20]$.

\subsection{Hochschild homology and cohomology of algebras}

For an associative $k$-algebra $A$, let $\bar{A}$ be its augmentation ideal. The reduced Hochschild chain complex of $A$ with coefficients in an $A$-bimodule $M$, denoted by $\mathrm{CH} \bullet(A ; M)$, is

$$
\cdots \rightarrow M \otimes \bar{A}^{\otimes n} \stackrel{b_{n}}{\longrightarrow} M \otimes \bar{A}^{\otimes n-1} \rightarrow \cdots \rightarrow M \otimes \bar{A} \stackrel{b_{1}}{\longrightarrow} M \rightarrow 0,
$$

with the boundary $b_{n}$ given by

$$
\begin{aligned}
b_{n}\left(m, \bar{a}_{1}, \ldots, \bar{a}_{n}\right)= & \left(m \bar{a}_{1}, \bar{a}_{2}, \ldots, \bar{a}_{n}\right)+\sum_{i=1}^{n-1}(-1)^{i}\left(m, \bar{a}_{1}, \ldots, \bar{a}_{i} \bar{a}_{i+1}, \ldots, \bar{a}_{n}\right) \\
& +(-1)^{n}\left(\bar{a}_{n} m, \bar{a}_{1}, \ldots, \bar{a}_{n-1}\right),
\end{aligned}
$$

for any $m \in M$ and $\bar{a}_{i} \in \bar{A}, i=1, \ldots, n$. The associated homology is called the Hochschild homology of $A$ with coefficients in $M$, and is denoted by $\mathrm{HH}_{\bullet}(A ; M)$.

The reduced Hochschild cochain complex $\mathrm{CH}^{\bullet}(A ; M)$ of $A$ with values in $M$ is the complex

$$
0 \rightarrow M \stackrel{\delta_{0}}{\longrightarrow} \operatorname{Hom}_{k}(\bar{A}, M) \stackrel{\delta_{1}}{\longrightarrow} \cdots \rightarrow \operatorname{Hom}_{k}\left(\bar{A}^{\otimes n}, M\right) \stackrel{\delta_{n}}{\longrightarrow} \cdots,
$$

with the coboundary $\delta_{n}$ given by

$$
\begin{aligned}
\left(\delta_{n} f\right)\left(\bar{a}_{1}, \ldots, \bar{a}_{n+1}\right)= & \bar{a}_{1} f\left(\bar{a}_{2}, \ldots, \bar{a}_{n+1}\right)+\sum_{i=1}^{n}(-1)^{i} f\left(\bar{a}_{1}, \ldots, \bar{a}_{i} \bar{a}_{i+1}, \ldots, \bar{a}_{n+1}\right) \\
& +(-1)^{n+1} f\left(\bar{a}_{1}, \ldots, \bar{a}_{n}\right) \bar{a}_{n+1},
\end{aligned}
$$

for any $f \in \operatorname{Hom}\left(\bar{A}^{\otimes n}, M\right)$ and $\bar{a}_{i} \in \bar{A}, i=1, \ldots, n+1$. The associated cohomology is called the Hochschild cohomology of $A$ with values in $M$, and is denoted by $\mathrm{HH}^{\bullet}(A ; M)$.

Later we will use the fact that $\operatorname{HH}_{n}(A ; M)=\operatorname{Tor}_{n}^{A^{e}}(A ; M)$ and $\operatorname{HH}^{n}(A ; M)=$ $\operatorname{Ext}_{A^{e}}^{n}(A ; M)$ (cf. [24, Lemma 9.1.3]). Let us recall the Connes cyclic operator on the Hochschild chain complex.

Definition 2.1 (Connes cyclic operator). For an associative algebra $A$, the Connes cyclic operator

$$
\mathrm{B}: \mathrm{CH}_{n}(A ; A) \rightarrow \mathrm{CH}_{n+1}(A ; A)
$$

is given by

$$
\mathrm{B}\left(a_{0}, \bar{a}_{1}, \ldots, \bar{a}_{n}\right)=\sum_{i=0}^{n}(-1)^{n i}\left(1, \bar{a}_{i}, \ldots, \bar{a}_{n}, \bar{a}_{0}, \ldots, \bar{a}_{i-1}\right) .
$$

It is easy to check $\mathrm{B}^{2}=\mathrm{B} b+b \mathrm{~B}=0$, and therefore $\left(\mathrm{CH}_{\bullet}(A ; A), b, \mathrm{~B}\right)$ is a mixed complex in the sense of Kassel [9].

Remark 2.2. The Hochschild homology and cohomology can also be defined for differential graded algebras. It is better to view these two homology groups as follows: 
Suppose $\left(A, d_{A}\right)$ is a possibly differential graded algebra and $\left(M, d_{M}\right)$ is a differential graded $A$-bimodule. Let $\mathrm{B}(A)$ be the bar construction of $A$ (see $[\mathbf{7}, \mathbf{1 6}, \mathbf{2 4}]$ for more details). Considering the following total complex

$$
\mathrm{CH}_{\bullet}(A ; M)=M \otimes \mathrm{B}(A),
$$

with the total degree of the tensor product, and the differential is $b=b_{0}+b_{1}$ given by

$$
b_{0}\left(m, \bar{a}_{1}, \ldots, \bar{a}_{n}\right)=-\left(d_{M} m, \bar{a}_{1}, \ldots, \bar{a}_{n}\right)-\sum_{i=1}^{n}(-1)^{\epsilon_{i-1}}\left(m, \bar{a}_{1}, \ldots, d_{A} \bar{a}_{i}, \ldots, \bar{a}_{n}\right)
$$

and

$$
\begin{aligned}
b_{1}\left(m, \bar{a}_{1}, \ldots, \bar{a}_{n}\right)= & -(-1)^{|m|}\left(m \bar{a}_{1}, \bar{a}_{2}, \ldots, \bar{a}_{n}\right)-\sum_{i=1}^{n-1}(-1)^{\epsilon_{i}}\left(m, \bar{a}_{1}, \ldots, \bar{a}_{i} \bar{a}_{i+1}, \ldots \bar{a}_{n}\right) \\
& +(-1)^{\left(\left|\bar{a}_{n}\right|-1\right) \epsilon_{n-1}}\left(\bar{a}_{n} m, \bar{a}_{1}, \ldots, \bar{a}_{n-1}\right),
\end{aligned}
$$

where $\epsilon_{i}=|m|+\left|a_{1}\right|+\cdots+\left|a_{i}\right|+i$, for any homogeneous elements $m \in M$ and $\bar{a}_{i} \in \bar{A}, i=1, \ldots n$. Here we denote by $\left|\bar{a}_{i}\right|$ the degree of $\bar{a}_{i}$. For $M=A$ and taking the gradings into account, the cyclic operator B can also be defined in the same way. For Hochschild cochain complex,

$$
\mathrm{CH}^{\bullet}(A ; M)=\operatorname{Hom}(\mathrm{B}(A), M),
$$

with the differential on the right-hand side analogously defined.

\subsection{Differential calculus with duality}

Let us recall the Gerstenhaber cup product and the bracket on the Hochschild cohomology of associative algebras, and its actions on the corresponding Hochschild homology of $A$.

Definition 2.3 (Gerstenhaber). A Gerstenhaber algebra is a graded $k$-vector space $A^{\bullet}$ endowed with two bilinear operators $\cup: A^{m} \otimes A^{n} \rightarrow A^{m+n}$ and $\{-,-\}: A^{n} \otimes$ $A^{m} \rightarrow A^{n+m-1}$ such that: for any homogeneous elements $a, b, c \in A^{\bullet}$,

(1) $\left(A^{\bullet}, \cup\right)$ is a graded commutative associative algebra, i.e.,

$$
a \cup b=(-1)^{|a||b|} b \cup a,
$$

satisfying associativity;

(2) $\left(A^{\bullet},\{-,-\}\right)$ is a graded Lie algebra with the bracket $\{-,-\}$ of degree -1 , i.e.,

$$
\{a, b\}=(-1)^{(|a|-1)(|b|-1)}\{b, a\}
$$

and

$$
\{a,\{b, c\}\}=\{\{a, b\}, c\}+(-1)^{(|a|-1)(|b|-1)}\{b,\{a, c\}\} ;
$$

(3) the cup product $\cup$ and the Lie bracket $\{-,-\}$ are compatible in the sense that

$$
\{a, b \cup c\}=\{a, b\} \cup c+(-1)^{(|a|-1)|b|} b \cup\{a, c\} .
$$

Definition 2.4 (Tamarkin-Tsygan [20, Definition 3.2.1]). Let $\mathrm{H}^{\bullet}$ and $\mathrm{H}_{\bullet}$ be two graded vector spaces. A differential calculus is a sextuple

$$
\left(\mathrm{H}^{\bullet}, \cup,\{-,-\}, \mathrm{H}_{\bullet}, \mathrm{B}, \cap\right),
$$


satisfying the following conditions

(1) $\left(\mathrm{H}^{\bullet}, \cup,\{-,-\}\right)$ is a Gerstenhaber algebra;

(2) $\mathrm{H}_{\bullet}$ is a graded module over $\left(\mathrm{H}^{\bullet}, \cup\right)$ by the "cap action"

$$
\cap: \mathrm{H}^{n} \otimes \mathrm{H}_{m} \rightarrow \mathrm{H}_{m-n}, f \otimes \alpha \mapsto f \cap \alpha,
$$

i.e. $(f \cup g) \cap \alpha=f \cap(g \cap \alpha)$ for any $f \in \mathrm{H}^{n}, g \in \mathrm{H}^{m}, \alpha \in \mathrm{H}_{s}$;

(3) there exists a linear operator $\mathrm{B}: \mathrm{H}_{\bullet} \rightarrow \mathrm{H}_{\bullet+1}$ such that $\mathrm{B}^{2}=0$ and, moreover, if we set $\mathrm{L}_{f}(\alpha):=\mathrm{B}(f \cap \alpha)-(-1)^{|f|} f \cap \mathrm{B}(\alpha)$, then

$$
\mathrm{L}_{\{f, g\}}(\alpha)=\left[\mathrm{L}_{f}, \mathrm{~L}_{g}\right](\alpha)
$$

and

$$
(-1)^{|f|+1}\{f, g\} \cap \alpha=\mathrm{L}_{f}(g \cap \alpha)-(-1)^{|g|(|f|+1)} g \cap\left(\mathrm{L}_{f}(\alpha)\right) .
$$

In the above definition, $\mathrm{L}_{f}(\alpha)$ is called the Lie derivative of $f$ on $\alpha$. It is shown by Daletskii, Gelfand and Tsygan in [6] that the Hochschild cohomology and homology of an associative algebra form a differential calculus. Let us give some details:

(1) The Gerstenhaber cup product $\cup: \mathrm{CH}^{n}(A ; A) \otimes \mathrm{CH}^{m}(A ; A) \rightarrow \mathrm{CH}^{n+m}(A ; A)$ is given by

$$
f \cup g\left(\bar{a}_{1}, \ldots, \bar{a}_{n+m}\right):=f\left(\bar{a}_{1}, \ldots, \bar{a}_{n}\right) g\left(\bar{a}_{n+1}, \ldots, \bar{a}_{n+m}\right) .
$$

(2) The Gerstenhaber Lie bracket

$$
\{-,-\}: \mathrm{CH}^{n}(A ; A) \otimes \mathrm{CH}^{m}(A ; A) \rightarrow \mathrm{CH}^{n+m-1}(A ; A)
$$

is given by

$$
\{f, g\}:=f \circ g-(-1)^{(|f|+1)(|g|+1)} g \circ f,
$$

where

$$
\begin{aligned}
& f \circ g\left(\bar{a}_{1}, \ldots, \bar{a}_{n+m-1}\right) \\
& \quad:=\sum_{i=0}^{n-1}(-1)^{(|g|+1) i} f\left(\bar{a}_{1}, \ldots, \bar{a}_{i}, \overline{g\left(\bar{a}_{i+1}, \ldots, \bar{a}_{i+m}\right)}, \bar{a}_{i+m+1}, \ldots, \bar{a}_{n+m-1}\right) .
\end{aligned}
$$

(3) The cap product $\cap: \mathrm{CH}^{n}(A ; A) \otimes \mathrm{CH}_{m}(A ; A) \rightarrow \mathrm{CH}_{m-n}(A ; A)$ is given by

$$
f \cap\left(a_{0}, \bar{a}_{1}, \ldots, \bar{a}_{m}\right):=\left(a_{0} f\left(\bar{a}_{1}, \ldots, \bar{a}_{n}\right), \bar{a}_{n+1}, \ldots, \bar{a}_{m}\right) .
$$

(4) The differential operator $\mathrm{B}$ on $\mathrm{CH}_{\bullet}(A ; A)$ is nothing but the Connes cyclic operator.

One can show that the above operations respect the boundary operators up to homotopy (which we will not address), and hence are well-defined on the homology level:

Proposition 2.5 (Daletskii-Gelfand-Tsygan [6]). Let $A$ be an associative algebra, then the data $\left(\mathrm{HH}^{\bullet}(A ; A), \cup,\{-,-\}, \mathrm{HH}_{\bullet}(A ; A), \mathrm{B}, \cap\right)$ forms a differential calculus.

\subsection{Another example of differential calculus}

Let $A^{!}$be a finite dimensional associative algebra and let $A^{\mathrm{i}}:=\operatorname{Hom}\left(A^{!}, k\right)$ be its dual space. Then $A^{\mathrm{i}}$ has an $A^{!}$-bimodule structure induced by the natural $A^{!}$-bimodule 
structure of $A^{!}$. There are two operators:

$$
\cap^{*}: \mathrm{CH}^{\bullet}\left(A^{!}\right) \times \mathrm{CH}^{\bullet}\left(A^{!} ; A^{\mathrm{i}}\right) \rightarrow \mathrm{CH}^{\bullet}\left(A^{!} ; A^{\mathrm{i}}\right)
$$

given by

$$
(f, \alpha) \mapsto f \cap^{*} \alpha=(-1)^{|f||\alpha|} \alpha \circ f
$$

and

$$
\mathrm{B}^{*}: \mathrm{CH}^{\bullet}\left(A^{!} ; A^{\mathrm{i}}\right) \rightarrow \mathrm{CH}^{\bullet}\left(A^{!} ; A^{\mathrm{i}}\right)
$$

given by

$$
\alpha \mapsto(-1)^{|\alpha|} \alpha \circ \mathrm{B} .
$$

Here $\mathrm{B}$ is the Connes cyclic operator on the Hochschild complex $\mathrm{CH}_{\bullet}\left(A^{!} ; A^{!}\right)$, and $\mathrm{CH}^{n}\left(A^{!} ; A^{\mathrm{i}}\right)$ is viewed as the linear dual space of $\mathrm{CH}_{n}\left(A^{!} ; A^{\mathrm{i}}\right)$ via the following identification

$$
\mathrm{CH}^{n}\left(A^{!} ; A^{\mathrm{i}}\right)=\operatorname{Hom}_{k}\left(\left(A^{!}\right)^{\otimes n}, A^{\mathrm{i}}\right) \cong \operatorname{Hom}_{k}\left(\left(A^{!}\right)^{\otimes n+1}, k\right) .
$$

Theorem 2.6. For a finite dimensional associative algebra $A^{!}$,

$$
\left(\mathrm{HH}^{\bullet}\left(A^{!}\right), \cup,\{-,-\}, \mathrm{HH}^{\bullet}\left(A^{!} ; A^{\mathrm{i}}\right), \mathrm{B}^{*}, \cap^{*}\right)
$$

forms a differential calculus.

Proof. A differential calculus is a pair of a Gerstenhaber algebra and a Gerstenhaber module. Since $\left(\mathrm{HH}^{\bullet}\left(A^{!}\right), \mathrm{HH}_{\bullet}\left(A^{!} ; A^{!}\right)\right)$forms a differential calculus and $\mathrm{HH}^{\bullet}\left(A^{!} ; A^{\mathrm{i}}\right)$ is the linear dual of $\mathrm{HH}_{\bullet}\left(A^{!} ; A^{!}\right)$, the Gerstenhaber module structure on $\mathrm{HH}^{\bullet}\left(A^{!} ; A^{\mathrm{i}}\right)$ is the adjoint of $\mathrm{HH}_{\bullet}\left(A^{!} ; A^{!}\right)$.

\subsection{The Batalin-Vilkovisky algebra structure}

In this paper we are mainly concerned with the Batalin-Vilkovisky algebra structure on the Hochschild cohomology.

Let us first recall the following notion of a differential calculus with duality, introduced by Lambre in $[\mathbf{1 3}]$.

Definition 2.7 (Lambre [13]). A differential calculus $\left(\mathrm{H}^{\bullet}, \cup,\{-,-\}, \mathrm{H}_{\bullet}, \mathrm{B}, \cap\right)$ is called a differential calculus with duality if there exists an integer $n$ and an isomorphism of $\mathrm{H}^{\bullet}$-modules

$$
\phi: \mathrm{H}^{\bullet} \rightarrow \mathrm{H}_{n-\bullet} .
$$

Lemma 2.8 (Lambre [13, Theorem 1.6 and Lemma 1.5]). Let $\left(\mathrm{H}^{\bullet}, \cup,\{-,-\}, \mathrm{H}_{\bullet}\right.$, $\mathrm{B}, \cap)$ be a differential calculus with duality and $\Delta:=\phi^{-1} \circ \mathrm{B} \circ \phi$. Then

$$
\{a, b\}=(-1)^{|a|+1}\left(\Delta(a \cup b)-\Delta(a) \cup b-(-)^{|a|} a \cup \Delta(b)\right) .
$$

The above lemma, in fact, says $\mathrm{H}^{\bullet}$ is a Batalin-Vilkovisky algebra. Let us recall its definition:

Definition 2.9. A Batalin-Vilkovisky algebra is a Gerstenhaber algebra $\left(\mathrm{H}^{\bullet}, \cup\right.$, $\{-,-\})$ together with an operator $\Delta: \mathrm{H}^{\bullet} \rightarrow \mathrm{H}^{\bullet-1}$ of degree -1 satisfying $\Delta \circ \Delta=0$, $\Delta(1)=0$ and

$$
\{a, b\}=(-1)^{|a|+1}\left(\Delta(a \cup b)-\Delta(a) \cup b-(-1)^{|a|} a \cup \Delta(b)\right),
$$

for any homogeneous elements $a, b \in \mathrm{H}^{\bullet}$. 


\section{Artin-Schelter regular algebras}

In this section, we briefly recall the construction of the Batalin-Vilkovisky algebra on the Hochschild cohomology of Artin-Schelter regular algebras with semisimple Nakayama automorphism, obtained by Kowalzig and Krahmer in [12]. In this section, $A$ is a connected graded algebra over an algebraically closed field $k$. A graded algebra $A$ is said to be connected if $A_{i}=0$ for $i<0$ and $A_{0}=k$.

Definition 3.1 (Artin-Schelter [1]). A connected graded algebra $A$ is called the Artin-Schelter regular (or AS-regular for short) of dimension $d$ if

(1) $A$ has finite global dimension $n$, and

(2) $A$ is Gorenstein, that is, $\operatorname{Ext}_{A}^{i}(k, A)=0$ for $i \neq n$ and $\operatorname{Ext}_{A}^{n}(k, A) \cong k$.

Later in 2014 Reyes, Rogalski and Zhang proved in [19] that AS-regular algebras are, in fact, twisted Calabi-Yau algebras (see also Yekutieli and Zhang [25] for some partial results):

Theorem 3.2 ([19, Lemma 1.2]). Let $A$ be a connected graded algebra. Then $A$ is $A S$ regular if and only if it is skew Calabi-Yau (in the graded sense), namely, A satisfies the following two conditions:

(1) A is homologically smooth, that is, A, viewed as an $A^{e}$-module, has a bounded, finitely generated projective resolution, and

(2) there exists an integer $n$ and an algebra automorphism $\sigma$ of $A$ such that

$$
\operatorname{Ext}_{A^{e}}^{i}(A, A \otimes A) \cong\left\{\begin{array}{cc}
0, & i \neq n \\
A_{\sigma}, & i=n
\end{array}\right.
$$

as $A^{e}$-modules.

In the above theorem, the automorphism $\sigma$ is called the Nakayama automorphism of $A$. The $A^{e}$-module structure of $\operatorname{Ext}_{A^{e}}^{d}(A, A \otimes A)$ is induced by the inner module structure on $A \otimes A: a \cdot(b \otimes c) \cdot d=b d \otimes a c$. The module $A_{\sigma}$ is a vector space $A$ equipped with the $A$-bimodule structure $a \cdot b \cdot c=a b \sigma(c)$, for any $a, b, c \in A$. We say $\sigma$ is semisimple if it is diagonalizable.

In the following, we will always use the above equivalent definition of AS-regular algebras, rather than its original definition.

\subsection{Results of Kowalzig and Krahmer}

Let $A$ be an AS-regular algebra with semi-simple Nakayama automorphism $\sigma$. In $[11, \mathbf{1 2}]$, a differential calculus with duality on $\left(\mathrm{HH}^{\bullet}(A), \mathrm{HH}_{\bullet}\left(A ; A_{\sigma}\right)\right)$ was constructed. Thus as a corollary, they obtained a Batalin-Vilkovisky algebra structure on $\mathrm{HH}^{\bullet}(A)$.

First, let us observe that, compared to the differential calculus structure given in $\S 2$, there is no Connes operator on $\mathrm{CH}_{\bullet}\left(A ; A_{\sigma}\right)$. They considered a subcomplex of $\mathrm{CH}_{\bullet}\left(A ; A_{\sigma}\right)$, whose homology is $\mathrm{HH}_{\bullet}\left(A ; A_{\sigma}\right)$ and on which the Connes operator is well-defined. Let us briefly recall their results.

Let

$$
\mathrm{B}: \mathrm{CH}_{p}\left(A ; A_{\sigma}\right) \rightarrow \mathrm{CH}_{p+1}\left(A ; A_{\sigma}\right)
$$


be given by

$$
\mathrm{B}\left(a_{0}, a_{1}, \ldots, a_{n}\right):=\sum_{i=0}^{n}(-1)^{n i}\left(1, a_{i}, \ldots, a_{n}, a_{0}, \sigma\left(a_{1}\right), \ldots, \sigma\left(a_{i-1}\right)\right)
$$

and

$$
\mathrm{T}: \mathrm{CH}_{p}\left(A ; A_{\sigma}\right) \rightarrow \mathrm{CH}_{p}\left(A ; A_{\sigma}\right)
$$

be given by

$$
\mathrm{T}\left(a_{0}, \ldots, a_{n}\right)=\left(\sigma\left(a_{0}\right), \ldots, \sigma\left(a_{n}\right)\right) .
$$

Lemma $3.3([\mathbf{1 2},(2.19)])$. Let $\mathrm{B}$ and $\mathrm{T}$ be as above, then there exists

$$
b \mathrm{~B}+\mathrm{B} b=\mathrm{Id}-\mathrm{T}
$$

on the complex $\mathrm{CH}_{\bullet}\left(A ; A_{\sigma}\right)$.

Since $\sigma$ is semisimple, there is a decomposition of $\mathrm{CH}_{\bullet}\left(A ; A_{\sigma}\right)$ as follows. Let $\Lambda$ be the set of eigenvalues of $\sigma$ acting on $A$ and $A_{\lambda}$ be the eigenvalue space corresponding to $\lambda \in \Lambda$. Denote

$$
\mathrm{CH}_{\bullet}^{\lambda}\left(A ; A_{\sigma}\right):=\bigoplus_{i} \bigoplus_{\prod_{j=1}^{i} \lambda_{i_{j}}=\lambda} A_{\lambda_{i_{1}}} \otimes \cdots \otimes A_{\lambda_{i_{i}}}, \quad \lambda_{i_{j}} \in \Lambda .
$$

The restriction of $b$ makes $\mathrm{CH}_{\bullet}^{\lambda}\left(A ; A_{\sigma}\right)$ to be a subcomplex of $\mathrm{CH}_{\bullet}\left(A ; A_{\sigma}\right)$ and we denote its homology by $\operatorname{HH}_{\bullet}^{\lambda}\left(A ; A_{\sigma}\right)$. A key observation is that, the restriction of $\mathrm{B}$ on the subcomplex $\mathrm{CH}_{\bullet}^{1}\left(A ; A_{\sigma}\right)$ is exactly the Connes cyclic operator. Hence $\left(\mathrm{CH}_{\bullet}^{1}\left(A ; A_{\sigma}\right), b, \mathrm{~B}\right)$ is a mixed complex.

As an immediate corollary of Lemma 3.3 , we have

$$
\mathrm{HH}_{\bullet}\left(A ; A_{\sigma}\right) \cong \mathrm{HH}_{\bullet}^{1}\left(A ; A_{\sigma}\right) \text {. }
$$

Via this isomorphism, we obtain the Connes operator $\mathrm{B}$ on $\mathrm{HH}_{\bullet}\left(A ; A_{\sigma}\right)$.

Similarly, there is a decomposition of the Hochschild cochain complex $\mathrm{CH}^{\bullet}(A ; A)$. Let

$$
\mathrm{CH}_{\mu}^{n}(A ; A):=\left\{f \in \mathrm{CH}^{n}(A ; A) \mid f\left(\bar{A}_{\mu_{1}} \otimes \cdots \otimes \bar{A}_{\mu_{m}}\right) \subset A_{\mu \mu_{1} \cdots \mu_{m}}\right\} .
$$

The restriction of coboundary $\delta$ makes $\mathrm{CH}_{\mu}^{\bullet}(A ; A)$ to be a subcomplex of $\mathrm{CH}^{\bullet}(A ; A)$ and we denote its cohomology by $\operatorname{HH}_{\mu}^{\bullet}(A ; A)$. In a similar fashion, they proved in [11] that the cohomology is concentrated in the subcomplex corresponding to the eigenvalue 1 , namely

$$
\mathrm{HH}^{\bullet}(A ; A) \cong \mathrm{HH}_{1}^{\bullet}(A ; A) .
$$

It is direct to check that the Gerstenhaber cup product, the bracket and the cap action restrict to the following maps: for $\lambda, \mu \in \Lambda$,

$$
\begin{aligned}
\cup: & \mathrm{CH}_{\lambda}^{p}(A ; A) \otimes \mathrm{CH}_{\mu}^{q}(A ; A) \rightarrow \mathrm{CH}_{\lambda \mu}^{p+q}(A ; A), \\
\{-,-\}: & \mathrm{CH}_{\lambda}^{p}(A ; A) \otimes \mathrm{CH}_{\mu}^{q}(A ; A) \rightarrow \mathrm{CH}_{\lambda \mu}^{p+q-1}(A ; A), \\
\cap: & \mathrm{CH}_{p}^{\lambda}\left(A ; A_{\sigma}\right) \otimes \mathrm{CH}_{\mu}^{q}(A ; A) \rightarrow \mathrm{CH}_{p-q}^{\lambda \mu}\left(A ; A_{\sigma}\right) .
\end{aligned}
$$

Considering the case of eigenvalues $\lambda=\mu=1$, we have the following theorem. 
Theorem 3.4 ([11, Theorem 1], [12, Theorem 1.5]). Let $\cup_{1}, \cap_{1}$ and $\{-,-\}_{1}$ be the restrictions of the cup product, the cap product and the Gerstenhaber bracket to the homology and cohomology spaces associated with the eigenvalue $\lambda=1$. Then together with the Connes operator $\mathrm{B}$, they give on

$$
\left(\mathrm{HH}_{1}^{\bullet}(A ; A), \cup_{1},\{-,-\}_{1}, \mathrm{HH}_{\bullet}^{1}\left(A ; A_{\sigma}\right), \mathrm{B}, \cap_{1}\right)
$$

a differential calculus structure.

The following is due to Van den Bergh [23, Proposition 2] (see also Brown-Zhang [4] for some further discussions):

Theorem 3.5. Let $A$ be an AS-regular algebra of finite global dimension $n$. Then we have the following isomorphism

$$
\mathrm{HH}_{n-\bullet}\left(A ; A_{\sigma}\right) \cong \mathrm{HH}^{\bullet}(A ; A) .
$$

Thus combining (2)-(4) we obtain the following:

Theorem 3.6 ([12, Theorem 4.25]). Let $A$ be an AS-regular algebra with semisimple Nakayama automorphism $\sigma$. Then

$$
\left(\mathrm{HH}^{\bullet}(A ; A), \cup,\{-,-\}, \mathrm{HH}_{\bullet}\left(A ; A_{\sigma}\right), \mathrm{B}, \cap\right)
$$

forms a differential calculus with duality.

Finally, the above theorem and Lemma 2.8 imply:

Theorem 3.7 ([12], Theorem 1.5). If $A$ is an AS-regular algebra with semisimple Nakayama automorphism, then the Hochschild cohomology $\mathrm{HH}^{\bullet}(A ; A)$ of $A$ is a Batalin-Vilkovisky algebra.

\section{Frobenius algebras}

In this section, we rephrase the construction of the Batalin-Vilkovisky algebra on the Hochschild cohomology of a Frobenius algebra with semisimple Nakayama automorphism, obtained by Lambre, Zhou and Zimmermann in [14].

Definition 4.1. A finite dimensional graded associative $k$-algebra $A^{!}$is called Frobenius of degree $n$ if there exists a nondegenerate bilinear pairing

$$
\langle-,-\rangle: A^{!} \otimes A^{!} \rightarrow k[n]
$$

such that $\langle a b, c\rangle=\langle a, b c\rangle$, for all $a, b, c \in A^{!}$.

Since the pairing is non-degenerate, there exists an automorphism $\sigma \in \operatorname{Aut}\left(A^{!}\right)$ such that $\langle a b, c\rangle=(-1)^{|c|(|a|+|b|)}\langle\sigma(c) a, b\rangle$. Such $\sigma$ is also called the Nakayama automorphism of $A^{!}$. The non-degeneracy of the pairing given by (5) is equivalent to saying that

$$
\psi: A^{!} \rightarrow A_{\sigma}^{\mathrm{i}}[n], \quad a \mapsto\langle-, a\rangle
$$

is an isomorphism of $A^{\text {! }}$-bimodules.

Let $A^{\mathrm{i}}=\operatorname{Hom}_{k}\left(A^{!}, k\right)$ be the linear dual space of $A^{!}$, which is a graded coalgebra. The Nakayama automorphism $\sigma$ induces an automorphism $\sigma^{*}$ on $A^{\mathrm{i}}$. Here $\sigma^{*}$ is the 
adjoint of $\sigma$. We have a left co-module structure on $A^{\mathrm{i}}$ :

$$
\Delta_{l}(a)=\sum_{(a)} \sigma^{*}\left(a^{\prime}\right) \otimes a^{\prime \prime},
$$

for all $a \in A^{\mathrm{i}}$. (The coproduct on $A^{\mathrm{i}}$ is viewed as a right co-module structure of $A^{\mathrm{i}}$, and is denoted by $\Delta_{r}(a)=\sum_{(a)} a^{\prime} \otimes a^{\prime \prime}$.) To distinguish, let us denote this new cobimodule structure on $A^{i}$ by $\sigma^{*} A^{i}$.

In $\S 2.3$ we obtain a differential calculus structure on $\left(\mathrm{HH}^{\bullet}\left(A^{!} ; A^{!}\right), \mathrm{HH}_{\bullet}\left(A^{!} ; A^{\mathrm{i}}\right)\right)$. In the following we will explore this structure in more detail, when $A^{!}$is a Frobenius algebra.

\subsection{Hochschild homology of coalgebras}

Suppose $C$ is a coassociative (possibly graded) coalgebra with coproduct $\Delta: C \rightarrow$ $C \otimes C$ given by

$$
\Delta(c)=\sum_{(c)} c^{\prime} \otimes c^{\prime \prime}:=c^{\prime} \otimes c^{\prime \prime}
$$

Let $\Delta^{0}:=\mathrm{Id}, \Delta^{1}:=\Delta$ and let $\Delta^{n}:=(\Delta \otimes i d \otimes \cdots \otimes i d) \circ \Delta^{n-1}$ by recursion. From the coassociativity of $\Delta$, we have $\Delta^{n}=(i d \otimes \cdots \otimes \Delta \otimes \cdots \otimes i d) \circ \Delta^{n-1}$.

Definition 4.2. Suppose $C$ is a coassociative (possibly graded) coalgebra and $M$ is a co-bimodule over $C$. The Hochschild chain complex of $C$ with coefficients in $M$, denoted by $\mathrm{CH}_{\bullet}(C ; M)$, is the complex

$$
0 \rightarrow M \rightarrow C \otimes M \rightarrow C^{\otimes 2} \otimes M \rightarrow \cdots \rightarrow C^{\otimes n} \otimes M \rightarrow \cdots,
$$

with the boundary $b$ given by

$$
\begin{aligned}
\delta^{*}\left(a_{1}, \ldots, a_{n}, m\right)= & \sum_{i}(-1)^{\left|a_{1}\right|+\cdots+\left|a_{i-1}\right|+i-1+\left|a_{i}^{\prime}\right|}\left(a_{1}, \ldots, a_{i}^{\prime}, a_{i}^{\prime \prime}, \ldots, a_{n}, m\right) \\
& +\sum_{(m)}(-1)^{\left|a_{1}\right|+\cdots+\left|a_{n}\right|+n+\left|c^{\prime}\right|}\left(\left(a_{1}, \ldots, a_{n}, c^{\prime}, m^{\prime}\right)\right. \\
& \left.+(-1)^{\epsilon}\left(c^{\prime \prime}, a_{1}, \ldots, a_{n}, m^{\prime \prime}\right)\right),
\end{aligned}
$$

where

$$
\Delta(m)=\sum_{(m)} c^{\prime} \otimes m^{\prime}+\sum_{(m)} m^{\prime \prime} \otimes c^{\prime \prime} \in C \otimes M \oplus M \otimes C,
$$

$\epsilon=\left(\left|c^{\prime \prime}\right|-1\right)\left(\left|a_{1}\right|+\cdots+\left|a_{n}\right|+n+\left|m^{\prime \prime}\right|\right)$ and $\left(a_{1}, \ldots, a_{n}, m\right) \in C^{\otimes n} \otimes M$. The associated homology is called the Hochschild homology of $C$ with coefficients in $M$, and is denoted by $\mathrm{HH}_{\bullet}(C ; M)$.

Theorem 4.3 ([14, Proposition 3.3]). Let $A^{!}$be a Frobenius algebra of degree $n$ with the Nakayama automorphism $\sigma$. Then there is an isomorphism

$$
\mathrm{PD}: \mathrm{HH}^{\bullet}\left(A^{!} ; A^{!}\right) \cong \mathrm{HH}_{n-\bullet}\left(A^{\mathrm{i}} ; \sigma^{*} A^{\mathrm{i}}\right) \text {. }
$$

We give a proof in the Koszul case.

Proof. Given a Frobenius algebra $A^{!}$with the Nakayama automorphism $\sigma$, we have $\psi: A^{!} \cong A_{\sigma}^{\mathrm{i}}[n]$ as $A^{!}$-bimodules. The $\psi$ is given by $\psi(a)=\langle-, a\rangle$. Therefore we have

$$
\operatorname{Hom}\left(\mathrm{B} A^{!}, A^{!}\right) \cong \operatorname{Hom}\left(\mathrm{B} A^{!}, A_{\sigma}^{\mathrm{i}}[n]\right) \cong \Omega\left(A^{\mathrm{i}}\right) \otimes A_{\sigma}^{\mathrm{i}}[n] \cong \Omega\left(A^{\mathrm{i}}\right) \otimes \sigma^{*} A^{\mathrm{i}}[n] .
$$


The isomorphisms above are all compatible with boundary maps, and hence we obtain

$$
\mathrm{PD}: \mathrm{HH}^{\bullet}\left(A^{!} ; A^{!}\right) \cong \mathrm{HH}_{n-\bullet}\left(A^{\mathrm{i}} ; \sigma^{*} A^{\mathrm{i}}\right) \text {. }
$$

\subsection{Frobenius algebra with semisimple Nakayama automorphism}

In this subsection, we go over the Batalin-Vilkovisky structure on the Hochschild cohomology of a Frobenius algebra with semisimple Nakayama automorphism.

Let us consider the Hochschild chain complex $\mathrm{CH}_{\bullet}\left(A^{\mathrm{i}} ; \sigma^{*} A^{\mathrm{i}}\right)$ of $A^{\mathrm{i}}$ with coefficients in $\sigma^{*} A^{\mathrm{i}}$. First, we define the map

$$
\mathrm{B}: \mathrm{CH}_{n}\left(A^{\mathrm{i}} ; \sigma^{*} A^{\mathrm{i}}\right) \rightarrow \mathrm{CH}_{n-1}\left(A^{\mathrm{i}} ; \sigma^{*} A^{\mathrm{i}}\right),
$$

given by

$$
\left(a_{1}, \ldots, a_{n}, a_{0}\right) \mapsto \sum_{i}(-1)^{i(n-i)} \epsilon\left(a_{0}\right)\left(a_{i+1}, \ldots, a_{n}, \sigma^{*}\left(a_{1}\right), \ldots, \sigma^{*}\left(a_{i-1}\right), a_{i}\right),
$$

where $\epsilon\left(a_{0}\right)$ is the image of the counit map $\epsilon: A^{\mathrm{i}} \rightarrow k$, and

$$
\mathrm{T}: \mathrm{CH}_{n}\left(A^{\mathrm{i}} ; \sigma^{*} A^{\mathrm{i}}\right) \rightarrow \mathrm{CH}_{n}\left(A^{\mathrm{i}} ; \sigma^{*} A^{\mathrm{i}}\right)
$$

given by

$$
\left(a_{1}, \ldots, a_{n}, a_{0}\right) \mapsto\left(\sigma^{*}\left(a_{1}\right), \ldots, \sigma^{*}\left(a_{n}\right), \sigma^{*}\left(a_{0}\right)\right) .
$$

Then we have the following

Lemma 4.4 ([14, Proposition 2.1], $[\mathbf{1 2},(2.19)])$. On the space $\mathrm{CH}_{\bullet}\left(A^{\mathrm{i}} ; \sigma^{*} A^{\mathrm{i}}\right)$, there exists the identity

$$
b \circ \mathrm{B}+\mathrm{B} \circ b=\mathrm{Id}-\mathrm{T} .
$$

Second, similar to the AS-regular case, there is a decomposition on the chain complex of a Frobenius coalgebra $A^{\mathrm{i}}$.

Since $\sigma$ is semisimple, there is a decomposition of $\mathrm{CH}_{\bullet}\left(A^{\mathrm{i}} ; \sigma^{*} A^{\mathrm{i}}\right)$ as follows. Let $\Lambda$ be the set of eigenvalues of $\sigma^{*}$ and $A_{\lambda}^{i}$ be the eigenvalue space corresponding to $\lambda \in \Lambda$. Let

$$
\mathrm{CH}_{\bullet}^{\lambda}\left(A^{\mathrm{i}} ; \sigma^{*} A^{\mathrm{i}}\right):=\bigoplus_{i} \bigoplus_{\prod_{j=1}^{i} \lambda_{i_{j}}=\lambda} A_{\lambda_{i_{1}}}^{\mathrm{i}} \otimes \cdots \otimes A_{\lambda_{i_{i}}}^{\mathrm{i}}, \quad \lambda_{i_{j}} \in \Lambda .
$$

The restriction of $d$ makes $\mathrm{CH}_{\bullet}^{\lambda}\left(A^{\mathrm{i}} ; \sigma^{*} A^{\mathrm{i}}\right)$ to be a subcomplex of $\mathrm{CH}_{\bullet}\left(A^{\mathrm{i}} ; \sigma^{*} A^{\mathrm{i}}\right)$ and we denote its homology by $\mathrm{HH}_{\bullet}^{\lambda}\left(A^{\mathrm{i}} ; \sigma^{*} A^{\mathrm{i}}\right)$. And the restriction map of $\mathrm{B}$ on the subcomplex $\mathrm{CH}_{\bullet}^{1}\left(A^{\mathrm{i}} ; \sigma A^{\mathrm{i}}\right)$ is exactly the Connes cyclic operator. Hence $\left(\mathrm{CH}_{\bullet}^{1}\left(A^{\mathrm{i}} ; \sigma^{*} A^{\mathrm{i}}\right), b, \mathrm{~B}\right)$ is a mixed complex.

Lemma 4.4 implies that

$$
\mathrm{HH}_{\bullet}\left(A^{\mathrm{i}} ; \sigma^{*} A^{\mathrm{i}}\right) \cong \mathrm{HH}_{\bullet}^{1}\left(A^{\mathrm{i}} ; \sigma^{*} A^{\mathrm{i}}\right) .
$$

There is also a decomposition of the Hochschild cochain complex $\mathrm{CH}^{\bullet}\left(A^{!} ; A^{!}\right)$. Let

$$
\mathrm{CH}_{\mu}^{n}\left(A^{!} ; A^{!}\right):=\left\{f \in \mathrm{CH}^{n}\left(A^{!} ; A^{!}\right) \mid f\left(\bar{A}_{\mu_{1}}^{!} \otimes \cdots \otimes \bar{A}_{\mu_{m}}^{!}\right) \subset A_{\mu \mu_{1} \cdots \mu_{m}}^{!}\right\} .
$$

The restriction of coboundary $b$ makes $\mathrm{CH}_{\mu}^{\bullet}\left(A^{!} ; A^{!}\right)$into a subcomplex of $\mathrm{CH}^{\bullet}\left(A^{!} ; A^{!}\right)$ and we denote its homology by $\operatorname{HH}_{\mu}^{\bullet}\left(A^{!} ; A^{!}\right)$. Similarly to $(3)$ we have

$$
\mathrm{HH}^{\bullet}\left(A^{!} ; A^{!}\right) \cong \mathrm{HH}_{1}^{\bullet}\left(A^{!} ; A^{!}\right) \text {. }
$$


It is direct to check that the Gerstenhaber cup product, the bracket and the cap product restrict to the following maps: for $\lambda, \mu \in \Lambda$,

$$
\begin{array}{rrrr}
\cup: & \mathrm{CH}_{\lambda}^{p}\left(A^{!} ; A^{!}\right) \otimes \mathrm{CH}_{\mu}^{q}\left(A^{!} ; A^{!}\right) & \rightarrow \mathrm{CH}_{\lambda \mu}^{p+q}\left(A^{!} ; A^{!}\right), \\
\{-,-\}: & \mathrm{CH}_{\lambda}^{p}\left(A^{!} ; A^{!}\right) \otimes \mathrm{CH}_{\mu}^{q}\left(A^{!} ; A^{!}\right) & \rightarrow \mathrm{CH}_{\lambda \mu}^{p+q-1}\left(A^{!} ; A^{!}\right), \\
\cap: & \mathrm{CH}_{p}^{\lambda}\left(A^{\mathrm{i}} ; \sigma^{*} A^{\mathrm{i}}\right) \otimes \mathrm{CH}_{\mu}^{q}\left(A^{!} ; A^{!}\right) & \rightarrow \mathrm{CH}_{p-q}^{\lambda \mu}\left(A^{\mathrm{i}} ; \sigma^{*} A^{\mathrm{i}}\right) .
\end{array}
$$

Considering the case of eigenvalues $\lambda=\mu=1$, we have the following theorem.

Theorem 4.5 ([14, Theorem 2.3]). Let $\cup_{1}, \cap_{1}$ and $\{-,-\}_{1}$ be the restrictions of the cup product, the cap product and the Gerstenhaber bracket to the homology and cohomology spaces associated with the eigenvalue $\lambda=1$. Then the Connes operator $\mathrm{B}$ gives on

$$
\left(\mathrm{HH}_{1}^{\bullet}\left(A^{!} ; A^{!}\right), \cup_{1},\{-,-\}_{1}, \mathrm{HH}_{\bullet}^{1}\left(A^{\mathrm{i}} ; \sigma^{*} A^{\mathrm{i}}\right), \mathrm{B}, \cap_{1}\right)
$$

a differential calculus structure.

Together with Theorem 4.3, we obtain the following.

Theorem 4.6 ([14, Theorem 2.3 and Proposition 3.4]). Suppose $A^{!}$is a Frobenius algebra of dimension d with semisimple Nakayama automorphism. Then

$$
\left(\mathrm{HH}^{\bullet}\left(A^{!} ; A^{!}\right), \cup,\{-,-\}, \mathrm{HH}_{\bullet}\left(A^{\mathrm{i}} ; \sigma^{*} A^{\mathrm{i}}\right), \mathrm{B}, \cap\right)
$$

forms a differential calculus with duality.

Combining the above theorem with Lemma 2.8, we obtain:

Theorem 4.7 ([14, Theorem 4.1]). If $A^{!}$is a Frobenius algebra with semisimple Nakayama automorphism, then the Hochschild cohomology $\mathrm{HH}^{\bullet}\left(A^{!} ; A^{!}\right)$of $A$ is a Batalin-Vilkovisky algebra.

\section{Koszul duality of AS-regular algebras}

In this section, we study Koszul AS-regular algebras, and then relate the two differential calculus structures in previous two sections by means of Koszul duality. We begin with Koszul algebras, which was introduced by Priddy in [17].

Assume $V$ is a $k$-vector space generated by a basis $\left\{x_{i}\right\}_{i=1}^{n}$ of degree 1 . The free algebra generated by $V$ is denoted by $\mathrm{T}(V)$. Let $R \subset V \otimes V$ be a subspace, and let $(R)$ be the two-sided ideal generated by $R$ in $\mathrm{T}(V)$. The quotient algebra $A=\mathrm{T}(V) /(R)$ is called a quadratic algebra.

Definition 5.1. Given a quadratic algebra $A=\mathrm{T}(V) /(R)$, the linear dual of $V$ is denoted by $V^{*}:=\operatorname{Hom}_{k}(V, k)$ and let $R^{\perp}:=\left\{f \in V^{*} \otimes V^{*}, f(r)=0, \forall r \in R\right\}$. Then $A^{!}:=\mathrm{T}\left(V^{*}\right) /\left(R^{\perp}\right)$ is called the quadratic dual of $A$.

Let $A^{\mathrm{i}}:=\operatorname{Hom}_{k}\left(A^{!}, k\right)$. Then

$$
A_{n}^{i} \cong \bigcap_{i+j+2=n}^{n}(s V)^{\otimes i} \otimes\left(s^{2} R\right) \otimes(s V)^{\otimes j},
$$

which is a coalgebra. Its coproduct is the restriction of the coproduct $\Delta$ on the co-free 
coalgebra $\mathrm{T}^{c}(s V)$ given by

$$
\Delta\left(a_{1}, \ldots, a_{n}\right)=\sum_{i=0}^{n}(-1)^{i}\left(a_{1}, \ldots, a_{i}\right) \otimes\left(a_{i+1}, \ldots, a_{n}\right) .
$$

Here the summand for $i=0$ has the form $1 \otimes\left(a_{1}, \ldots, a_{n}\right)$ and the summand for $i=n$ has the form $\left(a_{1}, \ldots, a_{n}\right) \otimes 1$.

The Koszul complex associated to $A$ is the complex

$$
0 \rightarrow A \otimes A_{n}^{\mathrm{i}} \stackrel{b}{\rightarrow} A \otimes A_{n-1}^{\mathrm{i}} \rightarrow \cdots \rightarrow A \otimes A_{1}^{\mathrm{i}} \stackrel{b}{\rightarrow} A \rightarrow k \rightarrow 0,
$$

with the differential $b$ given by

$$
b(a \otimes f)=\sum_{i} a x_{i} \otimes f x_{i}^{*}
$$

where $\left\{x_{i}^{*}\right\}_{i=1}^{n}$ is the dual basis of $V$ in $V^{*}$. It is direct to check $b^{2}=0$.

Definition 5.2. A quadratic algebra $A$ is called Koszul if the complex (6) is exact. In this case, $A^{!}$is called the Koszul dual algebra of $A$, and $A^{\mathrm{i}}$ is called the Koszul dual coalgebra of $A$.

One of the advantages of Koszul algebras is that $A$ has a much smaller free resolution, which is described as follows. Recall that the cobar construction $\Omega\left(A^{\mathrm{i}}\right)$ of $A^{\mathrm{i}}$ is the free tensor differential graded algebra generated by $s^{-1} \bar{A}^{i}$ with the differential $d$ given by

$$
\begin{aligned}
d_{\Omega(A \mathrm{i})}\left(s^{-1} a_{1}, \ldots, s^{-1} a_{n}\right) & \\
& =\sum_{i=1}^{n}(-1)^{\epsilon_{i}}\left(s^{-1} a_{1}, \ldots, s^{-1} a_{i-1}, s^{-1} a_{i}^{\prime}, s^{-1} a_{i}^{\prime \prime}, s^{-1} a_{i+1}, \ldots, s^{-1} a_{n}\right)
\end{aligned}
$$

for any $a_{i} \in \bar{A}^{i}, i=1, \ldots, n$, where $\epsilon_{i}=\left|a_{1}\right|+\cdots+\left|a_{i-1}\right|+i-1+\left|a_{i}^{\prime}\right|$.

Consider the composition of the following maps

$$
\left(A^{\mathrm{i}}\right)^{\otimes n} \stackrel{(p)^{\otimes n}}{\longrightarrow} V^{\otimes n} \longrightarrow A_{n}
$$

where $p: A^{\mathrm{i}} \rightarrow A_{1}^{\mathrm{i}}=V$ is the projection map and $V^{\otimes n} \rightarrow A_{n}$ is the natural surjective map. The composition map $q$ is denoted by

$$
q: \Omega\left(A^{\mathrm{i}}\right) \rightarrow A .
$$

For any $a_{k} \in A^{\mathrm{i}}, k=1, \ldots, n$, let

$$
q\left(a_{1}, \ldots, a_{n}\right)=\bar{a}_{1} \cdots \bar{a}_{n},
$$

where $\bar{a}_{i}$ is the image of the projection $p$.

Proposition 5.3 (Cf. [16, Theorem 3.4.4]). Let A be a Koszul algebra. Then

$$
q: \Omega\left(A^{\mathrm{i}}\right) \rightarrow A
$$

is a quasi-isomorphism.

Similarly, recall that $A_{m}^{\mathrm{i}}$ is a subset of $V^{\otimes m}$. Let $q^{\prime}: A_{m}^{\mathrm{i}} \rightarrow \bar{A}^{\otimes m}$ be the restriction map of the natural inclusion $V^{\otimes m} \rightarrow \bar{A}^{\otimes m}$, which extends to be a differential graded (DG) coalgebra map $\tilde{q}: A^{\mathrm{i}} \rightarrow \mathrm{B}(A)$. Then $\tilde{q}$ is also a quasi-isomorphism. 


\subsection{Homology of Koszul algebras with algebraic automorphisms}

Suppose $A$ is a Koszul algebra of global dimension $n$. Let $\sigma$ be an algebra automorphism of $A$ preserving the grading. Since $A_{1}=V$, we have $\sigma(V)=V$. Extending $\sigma$ to be an algebra map on $\mathrm{T}(V)$, we thus have $\sigma(R)=R$. This also means $\sigma$, by restriction on $A^{\mathrm{i}}$, is an automorphism of vector spaces.

Lemma 5.4. $\sigma$ is a coalgebra automorphism of $A^{i}$.

Proof. We need to prove

$$
(\sigma \otimes \sigma) \circ \Delta=\Delta \circ \sigma .
$$

Recall that $A_{n}^{\mathrm{i}} \cong \bigcap_{i+j+2=n} V^{\otimes i} \otimes R \otimes V^{\otimes j}$ and $\Delta$ is induced by the coproduct on the free tensor coalgebra $\mathrm{T}(V)$.

It is direct to check that $\sigma$ is a coalgebra map.

Consider the following complex

$$
0 \rightarrow A_{\sigma} \otimes A_{n}^{\mathrm{i}} \stackrel{b}{\rightarrow} A_{\sigma} \otimes A_{n-1}^{\mathrm{i}} \rightarrow \cdots \rightarrow A_{\sigma} \otimes A_{1}^{\mathrm{i}} \stackrel{b}{\rightarrow} A \rightarrow 0,
$$

with the differential $b$ given by

$$
b(a \otimes f)=a \sigma\left(x_{i}\right) \otimes x_{i}^{*} f+(-1)^{m} x_{i} a \otimes f x_{i}^{*},
$$

for any $a \otimes f \in A_{\sigma} \otimes A_{m}^{\mathrm{i}}$. It is easy to check $b^{2}=0$. We denote this complex by $\mathrm{K} \cdot\left(A_{\sigma}\right)=\left(A_{\sigma} \otimes A^{\mathrm{i}}, b\right)$.

Proposition 5.5. There is a quasi-isomorphism

$$
q: \mathrm{K}_{\bullet}\left(A_{\sigma}\right) \rightarrow\left(\mathrm{CH}_{\bullet}\left(A ; A_{\sigma}\right), b\right) .
$$

Proof. Consider the complex $\mathrm{K}_{\bullet}^{\prime}(A)=\bigoplus_{m} A \otimes A_{m}^{\mathrm{i}} \otimes A$ with the differential $b^{\prime}=$ $b_{L}^{\prime}+b_{R}^{\prime}$, where $b_{L}^{\prime}$ and $b_{R}^{\prime}$ are given by

$$
b_{L}^{\prime}(r \otimes f \otimes s)=\sum_{i} r x_{i} \otimes f x_{i}^{*} \otimes s, \quad b_{R}^{\prime}(r \otimes f \otimes s)=\sum_{i}(-1)^{m} r \otimes x_{i}^{*} f \otimes x_{i} s,
$$

for any $r \otimes f \otimes s \in \mathrm{K}_{m}(A)$. One can check that $\left(b_{L}^{\prime}\right)^{2}=\left(b_{R}^{\prime}\right)^{2}=b_{L}^{\prime} b_{R}^{\prime}+b_{R}^{\prime} b_{L}^{\prime}=0$. Hence $b^{2}=0$. The Koszul property of $A$ implies that $\mathrm{K}_{\bullet}^{\prime}(A)$ is a resolution of $A$ as an $A$-bimodule.

Now we have two $A$-bimodule free resolutions of $A, A \otimes A^{\mathrm{i}} \otimes A$ as above and the two-sided bar resolution $\tilde{\mathrm{B}}(A)$ (recall that it is $A \otimes \mathrm{B}(A) \otimes A$ with extra twisted differential). Recall that $A_{m}^{\mathrm{i}}$ is a subset of $V^{\otimes m}$. Let $q^{\prime}: A \otimes A^{\mathrm{i}} \otimes A \rightarrow A \otimes \mathrm{B}(A) \otimes A$ be the extension of $\tilde{q}$, which then commutes with the differentials on both sides. Then $q^{\prime}$ is a quasi-isomorphism (see [21, Proposition 3.3]).

It is clear that $A_{\sigma} \otimes_{A^{e}} \mathrm{~K}_{\bullet}^{\prime}(A)=\left(\mathrm{K}_{\bullet}\left(A_{\sigma}\right), b\right)$, and $A_{\sigma} \otimes_{A^{e}} \tilde{\mathrm{B}}(A)=\left(\mathrm{CH}_{\bullet}\left(A ; A_{\sigma}\right), b\right)$. Let $q=\operatorname{Id} \otimes q^{\prime}$. Then $q$ is a desired quasi-isomorphism.

\subsection{Two quasi-isomorphisms}

Suppose $A$ is a Koszul AS-regular algebra. The following result is well known.

Theorem 5.6 (Smith [18, Proposition 5.10]). Let $A$ be a Koszul algebra. Then $A$ is $A S$-regular if and only if $A^{!}$is Frobenius. 
Now suppose $A$ admits semisimple Nakayama automorphism $\sigma$. By [22] (Theorem 9.2), the adjoint $\sigma^{*}$ of $\sigma$ is the Nakayama automorphism of $A^{!}$. Since $\sigma$ is semisimple, $\sigma^{*}$ is also semisimple. Recall from previous subsection that $\sigma$ also gives semisimple Nakayama automorphism of $A^{i}$. The purpose of this subsection is to prove the following isomorphism

$$
\mathrm{HH}_{\bullet}\left(A ; A_{\sigma}\right) \cong \mathrm{HH}^{\bullet}\left(A^{!} ;{ }_{\sigma} A^{\mathrm{i}}\right)
$$

commutes with $\mathrm{B}$ on the left and $\mathrm{B}^{*}$ on the right.

Recall that the cobar construction $\Omega\left(A^{\mathrm{i}}\right)$ is a DG free algebra. We may extend the coalgebra automorphism $\sigma: A^{\mathrm{i}} \rightarrow A^{\mathrm{i}}$ to be a DG algebra automorphism of $\Omega\left(A^{\mathrm{i}}\right)$. Consider the complex $\mathrm{CH}_{\bullet}\left(\Omega\left(A^{\mathrm{i}}\right) ; \Omega\left(A^{\mathrm{i}}\right)_{\sigma}\right)$ (sometimes also denoted by $\Omega\left(A^{\mathrm{i}}\right)_{\sigma} \otimes$ $\left.\mathrm{B} \Omega\left(A^{\mathrm{i}}\right)\right)$. Assume $\sigma$ is semisimple, then we set $A_{\mu}^{\mathrm{i}}:=\left\{a \in A^{\mathrm{i}} \mid \sigma(a)=\mu a\right\}$. Let us denote

$$
\Omega\left(A^{\mathrm{i}}\right)_{\mu}=\bigoplus_{n \geqslant 0} \bigoplus_{\Pi_{i=1}^{n} \mu_{i}=\mu} A_{\mu_{1}}^{\mathrm{i}} \otimes \cdots \otimes A_{\mu_{n}}^{\mathrm{i}}
$$

and

$$
\mathrm{CH}_{\bullet}^{\mu}\left(\Omega\left(A^{\mathrm{i}}\right) ; \Omega\left(A^{\mathrm{i}}\right)_{\sigma}\right)=\bigoplus_{n \geqslant 0} \bigoplus_{\Pi_{i=0}^{n} \mu_{i}=\mu} \Omega\left(A^{\mathrm{i}}\right)_{\mu_{0}} \otimes \Omega\left(A^{\mathrm{i}}\right)_{\mu_{1}} \otimes \cdots \otimes \Omega\left(A^{\mathrm{i}}\right)_{\mu_{n}} .
$$

The restriction of $d$ makes $\mathrm{CH}_{\bullet}\left(\Omega\left(A^{\mathrm{i}}\right) ; \Omega\left(A^{\mathrm{i}}\right)_{\sigma}\right)_{\mu}$ into a complex. Denote its homology group by $\mathrm{HH}_{\bullet}^{\mu}\left(\Omega\left(A^{\mathrm{i}}\right), \Omega\left(A^{\mathrm{i}}\right)_{\sigma}\right)$. Again by Kowlzig and Krahmer [12, Proposition 2.7, Lemma 7.1] we have the following:

Lemma 5.7. On the complex

$$
\mathrm{CH}_{\bullet}\left(\Omega\left(A^{\mathrm{i}}\right) ; \Omega\left(A^{\mathrm{i}}\right)_{\sigma}\right),
$$

we have the identity

$$
d \circ \mathrm{B}+\mathrm{B} \circ d=\mathrm{Id}-\mathrm{T} .
$$

The above lemma implies

$$
\mathrm{HH}_{\bullet}\left(\Omega\left(A^{\mathrm{i}}\right) ; \Omega\left(A^{\mathrm{i}}\right)_{\sigma}\right) \cong \mathrm{HH}_{\bullet}^{1}\left(\Omega\left(A^{\mathrm{i}}\right) ; \Omega\left(A^{\mathrm{i}}\right)_{\sigma}\right),
$$

and the subcomplex $\mathrm{CH}_{\bullet}^{1}\left(\Omega\left(A^{\mathrm{i}}\right) ; \Omega\left(A^{\mathrm{i}}\right)_{\sigma}\right)$ is a mixed complex.

Lemma 5.8. Let $A$ be a Koszul algebra with an semi-simple automorphism $\sigma$, and $A^{\mathrm{i}}$ be its Koszul dual coalgebra. Then we have a commutative diagram of quasiisomorphisms of complexes up to homotopy

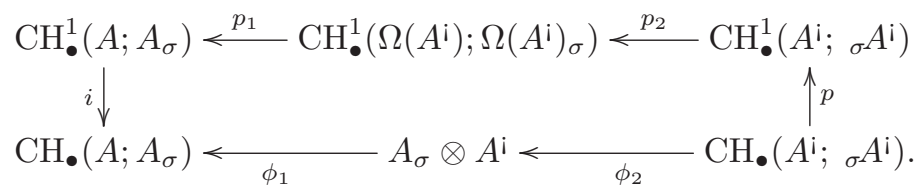

Proof. The map $p_{1}$ is defined by

$$
p_{1}:\left(\left(v_{1} \cdots v_{n}\right), a_{1}, \ldots, a_{m}\right) \mapsto\left(\left(\bar{v}_{1} \cdots \bar{v}_{n}\right), \bar{a}_{1}, \ldots, \bar{a}_{m}\right),
$$

here $a_{i}$ has the form $a_{i}:=\left(u_{i}^{1} \cdots u_{i}^{m_{i}}\right)$ with $a_{i}^{s} \in A^{\mathrm{i}}, s=1, \ldots, m_{i}$. And $\bar{a}_{i}$ is the image 
of $p: \Omega\left(A^{\mathrm{i}}\right) \rightarrow A$, that is, $p\left(u_{i}^{1} \cdots u_{i}^{m_{i}}\right)=\bar{u}_{i}^{1} \cdots \bar{u}_{i}^{m} \in A_{m}$. The map $p_{2}$ is given by

$$
p_{2}:\left(\left(v_{1} \cdots v_{n}\right), u\right) \mapsto\left(\left(v_{1} \cdots v_{n}\right),\left(u+\Delta(u)+\cdots+\Delta^{n}(u)+\cdots\right)\right),
$$

for $v_{i} \in A^{i}, i=0, \ldots, n$. At the bottom of the diagram, the map $\phi_{1}$ is given by

$$
\phi_{1}:\left(a,\left(v_{1} \cdots v_{m}\right)\right) \mapsto\left(a, v_{1}, \ldots, v_{m}\right),
$$

for $a \in A$ and $v_{i} \in V$. And the map $\phi_{2}$ is given by

$$
\phi_{2}:\left(v_{1}, \ldots, v_{n}, u\right) \mapsto\left(\bar{v}_{1} \cdots \bar{v}_{n}, u\right),
$$

for $v_{i} \in A^{\mathrm{i}}, i=0, \ldots, n$. In the vertical direction, $i$ is injection and $p$ is projection. They are quasi-isomorphisms up to homotopy. All these maps are all morphisms of complexes.

By a spectral sequence argument, all these morphisms are quasi-isomorphic. For example, let us consider $p_{1}$. There exist filtrations on these two complexes given by

$$
\mathrm{F}_{i}\left(\mathrm{CH}_{\bullet}^{1}\left(\Omega\left(A^{\mathrm{i}}\right) ; \Omega\left(A^{\mathrm{i}}\right)_{\sigma}\right)\right)=\bigoplus_{j \leqslant i}\left\{\left(a_{0}, a_{1}, \ldots, a_{j}\right) \mid a_{k} \in \Omega\left(A^{\mathrm{i}}\right), 0 \leqslant k \leqslant j\right\},
$$

and

$$
\mathrm{F}_{i}\left(\mathrm{CH}_{\bullet}^{1}\left(A ; A_{\sigma}\right)\right)=\bigoplus_{j \leqslant i}\left\{\left(b_{0}, b_{1}, \ldots, b_{j}\right) \mid b_{k} \in A, 0 \leqslant k \leqslant j\right\} .
$$

The boundary maps are compatible with the filtrations respectively. Then the comparison theorem for spectral sequences guarantees the quasi-isomorphism. Similarly we can prove other maps are quasi-isomorphisms.

Lemma 5.9. Let $A$ be a Koszul algebra with an semi-simple automorphism $\sigma$ and $A^{\mathrm{i}}$ be its Koszul dual coalgebra. Then we have the following quasi-isomorphisms of mixed complexes

$$
\mathrm{CH}_{\bullet}^{1}\left(A ; A_{\sigma}\right) \stackrel{p_{1} \mathrm{CH}_{\bullet}^{1}\left(\Omega\left(A^{\mathrm{i}}\right) ; \Omega\left(A^{\mathrm{i}}\right)_{\sigma}\right) q_{q_{2}}}{\longrightarrow} \mathrm{CH}_{\bullet}^{1}\left(A^{\mathrm{i} ;}{ }_{\sigma} A^{\mathrm{i}}\right),
$$

where $q_{2}$ is a homotopy inverse of $p_{2}$.

Proof. Since $\Omega\left(A^{\mathrm{i}}\right) \simeq A$ is a quasi-isomorphism of differential graded algebras, the map $p_{1}$ given in the previous lemma is a quasi-isomorphism of mixed complexes [15, Proposition 2.5.15]. We next construct the quasi-isomorphism $q_{2}$ of the mixed complexes, which is the homotopy inverse of $p_{2}$.

From now on, let us denote any homogeneous element in the bar construction $\mathrm{B} \Omega\left(A^{\mathrm{i}}\right)$ of $\Omega\left(A^{\mathrm{i}}\right)$ by $\left(a_{1}, \ldots, a_{n}\right)$ with $a_{i} \in \Omega\left(A^{\mathrm{i}}\right)$, and any homogeneous element in the cobar construction $\Omega\left(A^{\mathrm{i}}\right)$ of $A^{\mathrm{i}}$ by $\left(u_{1} \cdots u_{n}\right)$ or $\left(v_{1} \cdots v_{m}\right)$. The morphism $q_{2}$ is defined by

$$
\begin{aligned}
\mathrm{CH}_{\bullet}^{1}\left(\Omega\left(A^{\mathrm{i}}\right) ; \Omega\left(A^{\mathrm{i}}\right)_{\sigma}\right) & \rightarrow \mathrm{CH}_{\bullet}^{1}\left(A^{\mathrm{i}} ;{ }_{\sigma} A^{\mathrm{i}}\right), \\
\left(\left(u_{1} \cdots u_{n}\right), 1\right) & \mapsto\left(\left(u_{1} \cdots u_{n}\right), 1\right), \\
\left(\left(u_{1} \cdots u_{n}\right),\left(v_{1} \cdots v_{m}\right)\right) & \mapsto \sum_{i}(-1)^{\epsilon_{i}}\left(\left(v_{i+1} \cdots v_{m} u_{1} \cdots u_{n} \sigma\left(v_{1}\right) \cdots \sigma\left(v_{i-1}\right)\right), v_{i}\right), \\
\left(\left(u_{1} \cdots u_{n}\right), a_{1}, \ldots, a_{r}\right) & \mapsto 0, \quad r \geqslant 2,
\end{aligned}
$$

where $\epsilon_{i}=\left(\left|v_{i+1}\right|+\cdots+\left|v_{m}\right|-m+i\right)\left(\left|u_{1}\right|+\cdots+\left|u_{n}\right|-n+\left|v_{1}\right|+\cdots+\left|v_{i}\right|-i\right)$. 
It is clear $q_{2}$ is a morphism of complexes, that is, it commutes with the Hochschild differential. Now we show $q_{2}$ commutes with B. In fact,

(1) For Hochschild chains on the left hand side of (7), we have:

$\mathrm{B} \circ q_{2}\left(\left(u_{1} \cdots u_{n}\right), 1\right)=\mathrm{B}\left(\left(u_{1} \cdots u_{n}\right), 1\right)=\sum_{i=1}^{n}(-1)^{\epsilon_{i}}\left(u_{i+1} \cdots u_{n} \sigma\left(u_{1}\right) \cdots \sigma\left(u_{i-1}\right), u_{i}\right)$ and

$q_{2} \circ \mathrm{B}\left(\left(u_{1} \cdots u_{n}\right), 1\right)=q_{2}\left(1,\left(u_{1} \cdots u_{n}\right)\right)=\sum_{i=1}^{n}(-1)^{\epsilon_{i}}\left(u_{i+1} \cdots u_{n} \sigma\left(u_{1}\right) \cdots \sigma\left(u_{i-1}\right), u_{i}\right)$.

This means $q_{2} \circ \mathrm{B}=\mathrm{B} \circ q_{2}$ in this case.

(2) For Hochschild chains on the left hand side of (8), we have:

$$
\begin{aligned}
\mathrm{B} \circ q_{2}\left(\left(u_{1} \cdots u_{n}\right),\left(v_{1} \cdots v_{m}\right)\right) & =\mathrm{B}\left(\sum_{i=1}^{m}(-1)^{\epsilon_{i}} v_{i+1} \cdots v_{m} u_{1} \cdots u_{n} \sigma\left(v_{1}\right) \cdots \sigma\left(v_{i-1}\right), v_{i}\right) \\
& =0
\end{aligned}
$$

and

$$
q_{2} \circ \mathrm{B}\left(\left(u_{1} \cdots u_{n}\right),\left(v_{1} \cdots v_{m}\right)\right)=q_{2}(1, M)=0,
$$

for some $M \in \Omega\left(A^{\mathrm{i}}\right)^{\otimes 2}$. This means $q_{2} \circ \mathrm{B}=\mathrm{B} \circ q_{2}$ in this case.

(3) For Hochschild chains on the left hand side of $(9), q_{2} \circ \mathrm{B}=\mathrm{B} \circ q_{2}$ is automatic, since both sides are always zero.

In summary, the above calculation implies that $q_{2}$ is a morphism of mixed complexes. Next, we show that $p_{2}$ and $q_{2}$ are homotopy inverse to each other.

First, since

$$
\left.q_{2} \circ p_{2}\left(\left(u_{1} \cdots u_{n}\right), v\right)=q_{2}\left(\left(u_{1} \cdots u_{n}\right),(v+\Delta(v)+\cdots)\right)=\left(u_{1} \cdots u_{n}\right), v\right),
$$

we get $q_{2} \circ p_{2}=i d$ on $\Omega\left(A^{\mathrm{i}}\right)_{\sigma} \otimes A^{\mathrm{i}}$.

Second, we show

$$
p_{2} \circ q_{2} \simeq i d: \Omega\left(A^{\mathrm{i}}\right)_{\sigma} \otimes \mathrm{B} \Omega\left(A^{\mathrm{i}}\right) \rightarrow \Omega\left(A^{\mathrm{i}}\right)_{\sigma} \otimes \mathrm{B} \Omega\left(A^{\mathrm{i}}\right) .
$$

The homotopy map, denoted by $h$, is given as follows: First, let

$$
h_{0}(a)=0, \quad \text { for } a \in \Omega\left(A^{\mathrm{i}}\right),
$$

and for $n \geqslant 1$,

$$
h_{n}\left(a_{0}, a_{1}, \ldots, a_{n}, v\right)=0,
$$

and

$$
\begin{aligned}
h_{n}\left(a_{0}, a_{1}, \ldots, a_{n-1}, a_{n} v\right)= & (-1)^{\mu_{n}} h_{n}\left(v a_{0}, a_{1}, \ldots, a_{n-1}, a_{n}\right) \\
& +(-1)^{\nu_{n}} h_{n+1}\left(a_{0}, a_{1}, \ldots, a_{n}, v^{\prime} v^{\prime \prime}\right)+(-1)^{\epsilon_{n}}\left(a_{0}, a_{1}, \ldots, a_{n}, v\right),
\end{aligned}
$$

where $a_{i} \in \Omega\left(A^{\mathrm{i}}\right)$ for $i=0, \ldots, n$ and $v \in A^{\mathrm{i}}$. Here we use the Sweedler notation $\Delta(v)=\sum_{(v)} v_{(1)} \otimes v_{(2)}:=v^{\prime} \otimes v^{\prime \prime}$. The signs are given by

$$
\begin{gathered}
\mu_{n}=(|v|-1)\left(\left|a_{0}\right|+\cdots+\left|a_{n}\right|+n\right), \quad \nu_{n}=\left|a_{0}\right|+\cdots+\left|a_{n}\right|+n+\left|v^{\prime}\right|, \\
\epsilon_{n}=\left|a_{0}\right|+\cdots+\left|a_{n-1}\right|+n-1,
\end{gathered}
$$

where $\left|a_{i}\right|=\left|a_{i}^{1}\right|+\cdots+\left|a_{i}^{i_{s}}\right|-i_{s}, i=0, \ldots, n$ for any $a_{i}=\left(a_{i}^{1} \cdots a_{i}^{i_{s}}\right)$ with $a_{i}^{j} \in A^{i}$, 
$j=1, \ldots, i_{s}$. Now let $h=\sum_{i=0} h_{i}$ and we claim that

$$
h \circ d+d \circ h=i d-p_{2} \circ q_{2} .
$$

Assuming this identity, we obtain $q_{2}$ is a quasi-isomorphism of chain complexes, and thus a quasi-isomorphism of mixed complexes. This finishes our proof.

Proof of (10). For any element $\left(a_{0}, \ldots, a_{m}, v_{1} \cdots v_{n}\right) \in \Omega\left(A^{\mathrm{i}}\right)_{\sigma} \otimes \mathrm{B} \Omega\left(A^{\mathrm{i}}\right)$, where $a_{i}$ and $\left(v_{1} \cdots v_{m}\right) \in \Omega\left(A^{\mathrm{i}}\right)$, and $v_{i} \in A^{\mathrm{i}}$ for $i=1, \ldots, n$, we have

$$
h\left(a_{0}, \ldots, a_{n}, v_{1} \cdots v_{m}\right)=\sum_{i=2}^{m} \sum_{k=0}^{\infty}(-1)^{i_{k}}\left(v_{i+1} \cdots v_{m} a_{0}, \ldots, a_{n}, v_{1} \cdots v_{i-1}, \Delta^{k}\left(v_{i}\right)\right),
$$

where

$$
\begin{aligned}
i_{k}= & \mu_{m}+\cdots+\mu_{i+1}+\left|a_{0}\right|+\cdots+\left|a_{n}\right|+n+\left|v_{1}\right|+\cdots+\left|v_{i-1}\right|-i+1 \\
& +(k-1)\left|v_{i}^{(1)}\right|+\cdots+(k-i)\left|v_{i}^{(i)}\right|+\cdots+\left|v_{i}^{(k-1)}\right|
\end{aligned}
$$

and

$$
\mu_{s}=\left(\left|v_{s}\right|-1\right)\left(\left|a_{0}\right|+\cdots+\left|a_{n}\right|+n+\left|v_{1}\right|+\cdots+\left|v_{m}\right|-\left|v_{s}\right|-m+1\right),
$$

for $s=i+1, \ldots, m$, and where we write $\Delta^{k}(v)=v^{(1)} \otimes \cdots \otimes v^{(k+1)}$. We have the following three cases to check $h \circ d+d \circ h=i d-p_{2} \circ q_{2}$ :

(1) For Hochschild chains on the left hand side of (7), it is direct to see

$$
(h \circ d+d \circ h)\left(a_{0}, 1\right)=\left(i d-p_{2} \circ q_{2}\right)\left(a_{0}, 1\right)=0 .
$$

(2) For Hochschild chains on the left hand side of (8), we have

$$
d \circ h(a, w v)=\sum_{n=0}(-1)^{\epsilon_{n}} d\left(a, w, \Delta^{n}(v)\right),
$$

with $\epsilon_{n}=|a|+|w|+1+(n-1)\left|v^{(1)}\right|+\cdots+(n-k)\left|v^{(k)}\right|+\cdots+\left|v^{(n-1)}\right|$, where $a=$ $\left(u_{1} \cdots u_{m}\right)$ and $|a|=\left|u_{1}\right|+\cdots+\left|u_{m}\right|-m$, is equal to

$$
\begin{aligned}
& \sum_{n=0}(-1)^{\epsilon_{n}}\left(-(-1)^{|a|}\left(a \sigma(w), \Delta^{n}(v)\right)-(-1)^{|a|+|\omega|+1}\left(a, w v^{(1)}, v^{(2)}, \ldots, v^{(n)}\right)\right. \\
& -\sum_{s=1}^{n-1}(-1)^{|a|+|\omega|+\left|v^{(1)}\right|+\cdots+\left|v^{(s)}\right|+s+1}\left(a, w, v^{(1)}, \ldots, v^{(s)} v^{(s+1)}, \ldots, v^{(n)}\right) \\
& +(-1)^{\left|v^{(n)}-1\right|\left(|a|+|w|+\left|v^{(1)}+\cdots+\right| v^{(n-1)} \mid+n\right)}\left(v^{(n)} a, w, v^{(1)}, \ldots, v^{(n-1)}\right) \\
& +\sum_{i=1}^{m}(-1)^{\left|u_{1}\right|+\cdots+\left|u_{i-1}\right|-i+1+\left|u_{i}^{\prime}\right|+1}\left(\left(u_{1} \cdots u_{i}^{\prime} u_{i}^{\prime \prime} \cdots u_{m}\right), w, \Delta^{n}(v)\right) \\
& +(-1)^{|a|+\left|w^{\prime}\right|+1}\left(a, w^{\prime} w^{\prime \prime}, \Delta^{n}(v)\right) \\
& \left.+\sum_{i=1}^{n}(-1)^{|a|+|w|+\left|v^{(1)}\right|+\cdots+\left|v^{(i-1)}\right|+i+\left|v^{(i)^{\prime}}\right|+1}\left(a, w, v^{(1)}, \ldots, v^{(i)^{\prime}} v^{(i)^{\prime \prime}}, \ldots, v^{(n-1)}\right)\right) .
\end{aligned}
$$


The second summand

$$
\begin{aligned}
h \circ d(a, w v)= & \sum_{i=1}^{m}(-1)^{\left|u_{1}\right|+\cdots+\left|u_{i-1}\right|-i+\left|u_{i}^{\prime}\right|} h\left(\left(u_{1} \cdots u_{i}^{\prime} u_{i}^{\prime \prime} \cdots u_{m}\right), w v\right) \\
& +h\left(a,(-1)^{|a|+\left|w^{\prime}\right|+1} w^{\prime} w^{\prime \prime} v+(-1)^{|a|+|w|+\left|v^{\prime}\right|+1} w v^{\prime} v^{\prime \prime}\right)
\end{aligned}
$$

has the following terms (we omit the sign)

$$
\begin{aligned}
& (\Delta(a), w, v \pm \Delta(v)+\cdots) \pm(v a, \Delta(w) \pm \cdots) \pm(a, \Delta(w), v \pm \Delta(v) \pm \cdots) \\
& \pm\left(v^{\prime \prime} a, w, v^{\prime} \pm \Delta\left(v^{\prime}\right) \pm \cdots\right) \pm\left(a, w v^{\prime}, v^{\prime \prime} \pm \Delta\left(v^{\prime \prime}\right) \pm \cdots\right) .
\end{aligned}
$$

Recalling the bar and cobar construction and the corresponding differentials, we obtain that $h \circ d+d \circ d$ is equal to

$$
\begin{aligned}
\left(i d-p_{2} \circ q_{2}\right)(a, w v) & =(a, w v) \pm p_{2}(a \sigma(w), v) \pm p_{2}(v a, w) \\
& =(a, w v) \pm(a \sigma(w), v \pm \Delta(v) \pm \cdots) \pm(v a, w \pm \Delta(w) \pm \cdots) .
\end{aligned}
$$

The above identities imply that

$$
d \circ h+h \circ d=i d-p_{2} \circ q_{2} .
$$

For elements $\left(a, u_{1} \cdots u_{m}\right) \in \Omega\left(A^{i}\right)_{\sigma} \otimes \mathrm{B} \Omega\left(A^{\mathrm{i}}\right)$ with $m \geqslant 3$, it is easy to obtain the identity

$$
d \circ h+h \circ d=i d-p_{2} \circ q_{2}
$$

by similar computations as above.

(3) For Hochschild chains on the left hand side of (9)

$$
\left(a_{0}, \ldots, a_{k}, u_{1} \cdots u_{m}, v_{1} \cdots v_{n}\right) \in \Omega\left(A^{\mathrm{i}}\right)_{\sigma} \otimes \mathrm{B} \Omega\left(A^{\mathrm{i}}\right)
$$

where $a_{i},\left(u_{1} \cdots u_{m}\right)$ and $\left(v_{1} \cdots v_{n}\right) \in \Omega\left(A^{\mathrm{i}}\right)$, and $u_{i}, v_{j} \in A^{\mathrm{i}}$ for $i=1, \ldots, m, j=$ $1, \ldots, n$, we have $d \circ h\left(a_{0}, \ldots, a_{k}, u_{1} \cdots u_{m}, v_{1} \cdots v_{n}\right)$ equals

$$
\begin{aligned}
& \left(v_{i+1} \cdots \Delta\left(v_{j}\right) \cdots v_{m} a_{0}, a_{1}, \ldots, a_{k}, u_{1} \cdots u_{m}, v_{1} \cdots v_{i-1}, \Delta^{s}\left(v_{i}\right)\right) \\
& \pm\left(v_{i+1} \cdots v_{m} \Delta\left(a_{0}\right), a_{1}, \ldots, a_{k}, u_{1} \cdots u_{m}, v_{1} \cdots v_{i-1}, \Delta^{s}\left(v_{i}\right)\right) \\
& \pm\left(v_{i+1} \cdots v_{m} a_{0}, a_{1}, \ldots, \Delta\left(a_{j}\right), \ldots, a_{k}, u_{1} \cdots u_{m}, v_{1} \cdots v_{i-1}, \Delta^{s}\left(v_{i}\right)\right) \\
& \pm\left(v_{i+1} \cdots v_{m} a_{0}, a_{1}, \ldots, a_{k}, u_{1} \cdots \Delta\left(u_{j}\right) \cdots u_{m}, v_{1} \cdots v_{i-1}, \Delta^{s}\left(v_{i}\right)\right) \\
& \pm\left(v_{i+1} \cdots v_{m} a_{0}, a_{1}, \ldots, a_{k}, u_{1} \cdots u_{m}, v_{1} \cdots \Delta\left(v_{j}\right), v_{i-1}, \Delta^{s}\left(v_{i}\right)\right) \\
& \pm\left(v_{i+1} \cdots v_{m} a_{0}, a_{1}, \ldots, a_{k}, u_{1} \cdots u_{m}, v_{1} \cdots v_{i-1}, v_{i}^{(1)}, \ldots, v_{i}^{(j)^{\prime}} v_{i}^{(j)^{\prime \prime}}, \ldots, v_{i}^{(s+1)}\right) \\
& \pm\left(v_{i+1} \cdots v_{m} a_{0} \sigma\left(a_{1}\right), a_{2}, \ldots, a_{k}, u_{1} \cdots u_{m}, v_{1} \cdots v_{i-1}, \Delta^{s}\left(v_{i}\right)\right) \\
& \pm\left(v_{i+1} \cdots v_{m} a_{0}, a_{1}, \ldots, a_{j} a_{j+1}, \ldots, a_{k}, u_{1} \cdots u_{m}, v_{1} \cdots v_{i-1}, \Delta^{s}\left(v_{i}\right)\right) \\
& \pm\left(v_{i+1} \cdots v_{m} a_{0}, a_{1}, \ldots, a_{k-1} a_{k} u_{1} \cdots u_{m}, v_{1} \cdots v_{i-1}, \Delta^{s}\left(v_{i}\right)\right) \\
& \pm\left(v_{i+1} \cdots v_{m} a_{0}, a_{1}, \ldots, a_{k}, u_{1} \cdots u_{m} v_{1} \cdots v_{i-1}, \Delta^{s}\left(v_{i}\right)\right) \\
& \pm\left(v_{i+1} \cdots v_{m} a_{0}, a_{1}, \ldots, a_{k}, u_{1} \cdots u_{m}, v_{1} \cdots v_{i-1} v_{i}^{(1)}, v_{i}^{(2)}, \ldots, v_{i}^{(s+1)}\right) \\
& \pm\left(v_{i+1} \cdots v_{m} a_{0}, a_{1}, \ldots, a_{k}, u_{1} \cdots u_{m}, v_{1} \cdots v_{i-1}, v_{i}^{(1)}, \ldots, v_{i}^{(j)} v_{i}^{(j+1)}, \ldots, v_{i}^{(s+1)}\right) \\
& \pm\left(v_{i}^{(s+1)} v_{i+1} \cdots v_{m} a_{0}, a_{1}, \ldots, a_{k}, u_{1} \cdots u_{m}, v_{1} \cdots v_{i-1}, v_{i}^{(1)}, \ldots, v_{i}^{(s)}\right)
\end{aligned}
$$


and the second summand $h \circ d\left(a_{0}, a_{1}, \ldots, a_{k}, u_{1} \cdots u_{m}, v_{1} \cdots v_{n}\right)$ equals

$$
\begin{aligned}
& \left(v_{i+1} \cdots v_{m} a_{0} \sigma\left(a_{1}\right), a_{2}, \ldots, a_{k}, u_{1} \cdots u_{m}, v_{1} \cdots v_{i-1}, \Delta^{s}\left(v_{i}\right)\right) \\
& \pm\left(v_{i+1} \cdots v_{m} a_{0}, a_{1}, \ldots, a_{j} a_{j+1}, \ldots, a_{k}, u_{1} \cdots u_{m}, v_{1} \cdots v_{i-1}, \Delta^{s}\left(v_{i}\right)\right) \\
& \pm\left(v_{i+1} \cdots v_{m} a_{0}, a_{1}, \ldots, a_{k-1} a_{k} u_{1} \cdots u_{m}, v_{1} \cdots v_{i-1}, \Delta^{s}\left(v_{i}\right)\right) \\
& \pm\left(v_{i+1} \cdots v_{m} a_{0}, a_{1}, \ldots, a_{k}, u_{1} \cdots u_{m} v_{1} \cdots v_{i-1}, \Delta^{s}\left(v_{i}\right)\right) \\
& \pm\left(u_{i+1} \cdots u_{m} v_{1} \cdots v_{n} a_{0}, a_{1}, \ldots, a_{k}, u_{1} \cdots u_{i-1}, \Delta^{s}\left(u_{i}\right)\right) \\
& \pm\left(u_{i+1} \cdots u_{m} v_{1} \cdots v_{n} a_{0}, a_{1}, \ldots, a_{k}, u_{1} \cdots u_{i-1}, \Delta^{s}\left(u_{i}\right)\right) \\
& \pm\left(v_{i+1} \cdots v_{m} \Delta\left(a_{0}\right), a_{1}, \ldots, a_{k}, u_{1} \cdots u_{m}, v_{1} \cdots v_{i-1}, \Delta^{s}\left(v_{i}\right)\right) \\
& \pm\left(v_{i+1} \cdots v_{m} a_{0}, a_{1}, \ldots, \Delta\left(a_{j}\right), \ldots, a_{k}, u_{1} \cdots u_{m}, v_{1} \cdots v_{i-1}, \Delta^{s}\left(v_{i}\right)\right) \\
& \pm\left(v_{i+1} \cdots v_{m} a_{0}, a_{1}, \ldots, a_{k}, u_{1} \cdots \Delta\left(u_{j}\right) \cdots u_{m}, v_{1} \cdots v_{i-1}, \Delta^{s}\left(v_{i}\right)\right) \\
& \left. \pm\left(v_{i+1} \cdots v_{j}\right) \cdots v_{m} a_{0}, a_{1}, \cdots, a_{k}, u_{1} \cdots u_{m}, v_{1} \cdots v_{i-1}, \Delta^{s}\left(v_{i}\right)\right) \\
& \pm\left(v_{i}^{\prime \prime} v_{i+1} \cdots v_{m} a_{0}, a_{1}, \ldots, a_{k}, u_{1} \cdots u_{m}, v_{1} \cdots v_{i-1}, \Delta^{s}\left(v_{i}^{\prime}\right)\right) \\
& \pm\left(v_{i+1} \cdots v_{m} a_{0}, a_{1}, \ldots, a_{k}, u_{1} \cdots u_{m}, v_{1} \cdots v_{i-1} v_{i}^{\prime}, \Delta^{s-1}\left(v_{i}^{\prime \prime}\right)\right) \\
& \pm\left(v_{i+1} \cdots v_{m} a_{0}, a_{1}, \ldots, a_{k}, u_{1} \cdots u_{m}, v_{1} \cdots \Delta\left(v_{j}\right) \cdots v_{i-1}, \Delta^{s-1}\left(v_{i}\right)\right),
\end{aligned}
$$

where $\Delta\left(a_{i}\right)=\sum_{s=1}^{n_{i}}\left(a_{i}^{1} \cdots a_{i}^{s-1} a_{i}^{s^{\prime}} a_{i}^{s^{\prime \prime}} a_{i}^{s+1} \cdots a_{i}^{n_{i}}\right)$ if $a_{i}$ has the form $a_{i}=\left(a_{i}^{1} \cdots a_{i}^{n_{i}}\right)$ with $a_{i}^{j} \in A^{\mathrm{i}}, j=0, \ldots, n_{i}$. From the coassociativity of the coproduct $\Delta$ on $A^{\mathrm{i}}$, we see that the sum of the above two expressions

$(d \circ h+h \circ d)\left(a_{0}, a_{1}, \ldots, a_{k}, u_{1} \cdots u_{m}, v_{1} \cdots v_{n}\right)=\left(a_{0}, a_{1}, \ldots, a_{k}, u_{1} \cdots u_{m}, v_{1} \cdots v_{n}\right)$.

Since $q_{2}\left(a_{0}, a_{1}, \ldots, a_{k}, u_{1} \cdots u_{m}, v_{1} \cdots v_{n}\right)=0$ for $k \geqslant 1$, we have the identity

$$
d \circ h+h \circ d=i d-p_{2} \circ q_{2} .
$$

In summary, we have proved the desired identity.

Theorem 5.10. Let $A$ be a Koszul AS-regular algebra with semisimple Nakayama automorphism $\sigma$. Then we have isomorphisms

$$
\mathrm{HH}_{\bullet}\left(A ; A_{\sigma}\right) \cong \mathrm{H}_{\bullet}\left(\mathrm{K}_{\bullet}\left(A_{\sigma}\right)\right) \cong \mathrm{HH}_{\bullet}\left(A^{\mathrm{i}} ;{ }_{\sigma} A^{\mathrm{i}}\right)
$$

and these isomorphisms respect the Connes operator on both sides.

Proof. Combining the above two lemmas and the following fact

$$
\left(A_{\sigma} \otimes A^{\mathrm{i}}, b\right) \cong\left(A \otimes{ }_{\sigma} A^{\mathrm{i}}, b\right) \simeq\left(\Omega\left(A^{\mathrm{i}}\right) \otimes{ }_{\sigma} A^{\mathrm{i}}, \delta^{*}\right),
$$

where we use the $A$-bimodule structure of $A_{\sigma}$ in $A_{\sigma} \otimes A^{\mathrm{i}}$ and the $A^{\mathrm{i}}$-cobimodule structure of ${ }_{\sigma} A^{\mathrm{i}}$ in $A \otimes{ }_{\sigma} A^{\mathrm{i}}$, we get the proof.

Now consider the following complex

$$
\mathrm{K}^{\bullet}\left(A \otimes A^{!}\right)=\left\{0 \rightarrow A \otimes A \rightarrow \cdots \rightarrow A \otimes A_{n-1}^{!} \otimes A \rightarrow A \otimes A_{n}^{!} \otimes A \rightarrow \cdots\right\},
$$

with coboundary $\delta$ given by

$$
\delta(a \otimes f \otimes b):=a \otimes x_{i}^{*} f \otimes x_{i} b+(-1)^{m} a x_{i} \otimes f x_{i}^{*} \otimes b .
$$


Theorem 5.11. Let $A$ be a Koszul algebra. Then there are natural isomorphisms

$$
\mathrm{HH}^{\bullet}(A) \cong \mathrm{H}^{\bullet}\left(\mathrm{K}^{\bullet}\left(A \otimes A^{!}\right)\right) \cong \mathrm{HH}^{\bullet}\left(A^{!}\right)
$$

of graded commutative algebras. The products on both sides are the Gerstenhaber cup products.

Proof. See Buchweitz [2], or Beilinson-Ginzburg-Soergel [3], or Keller [10].

\subsection{Proof of the main theorem}

We are now ready to prove the main theorem of this paper.

Proof of Theorem 1.1. By Theorems 3.5 and 4.3 we have the following commutative diagram

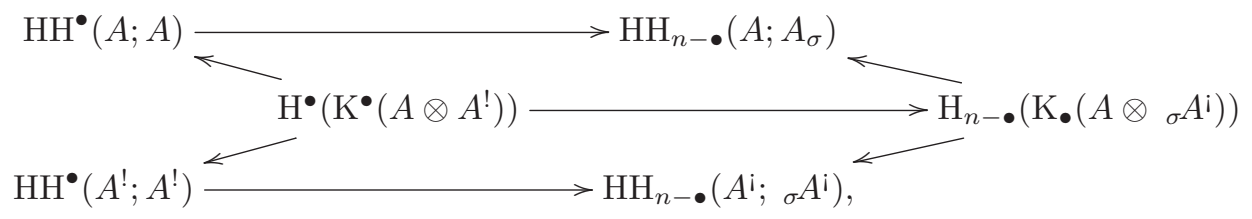

which gives the following commutative diagram

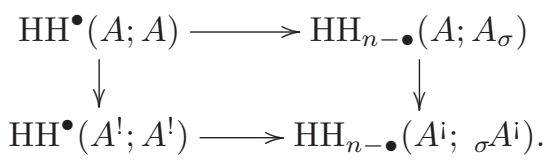

Theorem 5.11 says that the left vertical map is an isomorphism of graded algebras, and Theorem 5.10 says that the right vertical map respects the Connes operators. Thus by Lemma 2.8 we see that two Batalin-Vilkovisky algebras are isomorphic.

\section{References}

[1] M. Artin and W.F. Schelter, Graded algebras of global dimension 3, Adv. Math. 66(1987), 171-216.

[2] R.-O. Buchweitz, Hochschild cohomology of Koszul algebras, talk at the Conference on Representation Theory, Canberra, July 2003.

[3] A. Beilinson, V. Ginzburg and W. Soergel, Koszul duality patterns in representation theory, J. Amer. Math. Soc. 9(1996), 473-527.

[4] K.A. Brown and J.J. Zhang, Dualizing complexes and twisted Hoschild (co)homology for Noetherian Hopf algebras, J. Algebra 320(2008), 1814-1850.

[5] X. Chen, S. Yang and G.D. Zhou, Batalin-Vilkovisky algebras and noncommutative Poincare duality of Koszul Calabi-Yau algebras, J. Pure Appl. Algebra 220(2016), 2500-2532.

[6] Y. Daletskii, I. Gelfand and B. Tsygan, On a variant of noncommutative differential geometry, Dokl. Akad. Nauk SSSR 308(1989), 1293-1297 (in Russian), translation in Soviet Math. Dokl. 40(1990), 422-426.

[7] E. Getzler, J. Jones and S. Petrack, Differential forms on loop spaces and the cyclic bar complex, Topology 30(1991), 339-371. 
[8] V. Ginzburg, Calabi Yau algebras, arXiv: 0612139v3.

[9] C. Kassel, L'homologie cyclique des algebres enveloppantes, Invent. Math. 91(1988), 221-251.

[10] B. Keller, Derived invariance of higher structures on the Hochschild complex, available at http://www.math.jussieu.fr/ keller/publ/dihabs.html.

[11] N. Kowalzig and U. Krahmer, Duality and products in algebraic (co)homology theories, J. Algebra 323(2010), 2063-2081.

[12] N. Kowalzig and U. Krahmer, Batalin-Vilkovisky structures on Ext and Tor, J. Reine Angew. Math. 697(2014), 159-219.

[13] T. Lambre, Dualité de Van den Bergh et Structure de Batalin-Vilkovisky sur les algèbres de Calabi-Yau, J. Noncom. Geom. 3(2010) 441-457.

[14] T. Lambre, G.D. Zhou and A. Zimmermann, The Hochschild cohomology ring of a Frobenius algebra with semisimple Nakayama automorphism is a BatalinVilkovisky algebra, J. Algebra 446(2016), 103-131.

[15] J.-L. Loday, Cyclic homology, 2nd edition, Grundlehren Math. Wiss. 301, Springer-Verlag, Berlin, 1998.

[16] J.-L. Loday and B. Vallete, Algebraic operad, Grundlehren Math. Wiss. 346, Springer, Heidelberg, 2012.

[17] S.B. Priddy, Koszul resolutions, Trans. Amer. Math. Soc. 152(1970), 39-60.

[18] S. Paul Smith, Some finite dimensional algebras related to elliptic curves, Representation theory of algebras and related topics (Mexico City, 1994), 315-348, CMS Conf. Proc. 19, Amer. Math. Soc., Providence, RI, 1996.

[19] M. Reyes, D. Rogalski and J.J. Zhang, Skew Calabi-Yau algebras and Homological identities, Adv. Math. 264(2014), 308-354.

[20] D. Tamarkin and B. Tsygan, The ring of differential forms in noncommutative calculus, Proc. Sympos. Pure Math. 73, Amer. Math. Soc., Providence, RI, 2005.

[21] M. Van den Bergh, Noncommutative homology of some three-dimensional quantum space, K-Theory 8(1994), 213-230.

[22] M. Van den Bergh, Existence theorems for dualizing complexes over noncommutative graded and filtered rings, J. Algebra 195(1997), 662-679.

[23] M. Van den Bergh, A relation between Hochschild homology and cohomology for Gorenstein algebras, Proc. Amer. Math. Soc. 126(1998), 1345-1348, and Erratum, Proc. Amer. Math. Soc. 130(2002), 2809-2810.

[24] C. Weibel, An introduction to homological algebra, Cambridge Stud. Adv. Math. 38, Cambridge University Press, London, 1994.

[25] A. Yukutieli and J.J. Zhang, Homological transcendence degree, Proc. London Math. Soc. 93(2006), 105-137.

Leilei Liu liuleilei199009@126.com

Department of Mathematics, Sichuan University, Chengdu, Sichuan Province 610064, People's Republic of China 